\title{
EXISTENCE, UNIQUENESS AND CONVERGENCE OF A PARTICLE APPROXIMATION FOR THE ADAPTIVE BIASING FORCE PROCESS*
}

\author{
Benjamin Jourdain ${ }^{1}$, Tony Lelièvre ${ }^{2}$ and RaphaËL Roux ${ }^{2}$
}

\begin{abstract}
We study a free energy computation procedure, introduced in [Darve and Pohorille, J. Chem. Phys. 115 (2001) 9169-9183; Hénin and Chipot, J. Chem. Phys. 121 (2004) 2904-2914], which relies on the long-time behavior of a nonlinear stochastic differential equation. This nonlinearity comes from a conditional expectation computed with respect to one coordinate of the solution. The long-time convergence of the solutions to this equation has been proved in [Lelièvre et al., Nonlinearity 21 (2008) 1155-1181], under some existence and regularity assumptions. In this paper, we prove existence and uniqueness under suitable conditions for the nonlinear equation, and we study a particle approximation technique based on a Nadaraya-Watson estimator of the conditional expectation. The particle system converges to the solution of the nonlinear equation if the number of particles goes to infinity and then the kernel used in the Nadaraya-Watson approximation tends to a Dirac mass. We derive a rate for this convergence, and illustrate it by numerical examples on a toy model.
\end{abstract}

Mathematics Subject Classification. 60H10, 60K35, 65C35, 82C31.

Received February 24, 2009.

Published online August 26, 2010.

\section{INTRODUCTION}

Free energy computations are an important problem in the field of molecular simulation (see [4]). The difficulty of those computations lies in the fact that most dynamics in molecular simulations are highly metastable: many free energy barriers prevent a good sampling. We study here the adaptive biasing force (ABF) method, which was introduced in $[5,8]$ to get rid of those metastabilities.

The typical problems one can think about are the study of a structural angle in the conformation of a protein, or the measure of the evolution of a chemical reaction. Mathematically, each configuration of the system is modelized by an element of a high-dimensional state space $\mathcal{D}$, typically an open subset of $\mathbb{R}^{d}$, which is endowed with a probability measure, called the canonical measure. This measure is given by $\left(\int_{\mathcal{D}} \mathrm{e}^{-\beta V(x)} \mathrm{d} x\right)^{-1} \mathrm{e}^{-\beta V(x)} \mathrm{d} x$, where $V$ denotes the potential energy undergone by the physical system, and $\beta$ is proportional to the inverse of the temperature of the system.

Keywords and phrases. Conditional McKean nonlinearity, interacting particle systems, Adaptive Biasing Force method.

* This work was supported by the french National Research Agency (ANR) under the programs ANR-08-BLAN-0218-03 BigMC and $A N R-09-B L A N M E G A S$.

1 Université Paris-Est, CERMICS, 6 et 8 avenue Blaise Pascal, 77455 Marne-La-Vallée Cedex 2, France.

2 Université Paris-Est, CERMICS, Project-Team MICMAC ENPC-INRIA, 6 et 8 avenue Blaise Pascal, 77455 Marne-La-Vallée Cedex 2, France. lelievre@cermics.enpc.fr 
For some $x$ in the state space, one is interested in a particular quantity, denoted by $\xi(x), \xi$ being assumed to be a smooth function from $\mathcal{D}$ to the one-dimensional torus $\mathbb{T}$. The quantity $\xi(x)$ has to be understood as a coarse-grained information on the system, which is the relevant information for the practitioner. In the examples above, $\xi(x)$ would be a structural angle in a protein with conformation $x$, or a number measuring the evolution of a chemical system in state $x$.

We call free energy the effective energy associated to the quantity $\xi(x)$, that is, the function $A(z)$ such that $\mathrm{e}^{-\beta A(z)} \mathrm{d} z$ is the image measure of the canonical measure by the function $\xi$. Our objective is to compute numerically the function $A$. When $\mathcal{D}=\mathbb{R}^{d}$, a naive method to do so is to simulate, for a given random variable $X_{0}$ and an independent $\mathbb{R}^{d}$-valued Brownian motion $W$, the process defined by the (overdamped) Langevin dynamics

$$
\mathrm{d} X_{t}=-\nabla V\left(X_{t}\right) \mathrm{d} t+\sqrt{2 \beta^{-1}} \mathrm{~d} W_{t}
$$

which, under some regularity assumptions on the potential, is ergodic and admits the canonical measure as unique invariant measure. This approach appears to be untractable in practice, since the convergence to equilibrium is very slow, due to multiple metastabilities appearing in most problems: typically, a molecule moves microscopically within times of order $10^{-15} \mathrm{~s}$, while the typical time scale of the macroscopic moves is of order $10^{-9} \mathrm{~s}$.

The idea of the ABF method is to prevent the process $X_{t}$ from staying in metastable states by introducing a biasing force which repel $X_{t}$ from the states where it stayed for too long a time. To do this, we use the following representation of $A$, that can be deduced from the co-area formula (see [11]):

$$
A^{\prime}(z)=\mathbb{E}[F(X) \mid \xi(X)=z],
$$

where $X$ is a random variable distributed according to the canonical measure, and $F$ is the function defined by

$$
F(x)=\frac{\nabla \xi \cdot \nabla V}{|\nabla \xi|^{2}}-\frac{1}{\beta} \operatorname{div}\left(\frac{\nabla \xi}{|\nabla \xi|^{2}}\right) .
$$

The function $A^{\prime}$ is called the mean force. Actually, (1.2) also holds when $X$ is distributed according to the measure

$$
\left(\int_{\mathcal{D}} \mathrm{e}^{-\beta(V(x)+W \circ \xi(x))} \mathrm{d} x\right)^{-1} \mathrm{e}^{-\beta(V(x)+W \circ \xi(x))} \mathrm{d} x,
$$

which is the canonical measure associated with the biased potential $V+W \circ \xi$ where $W$ is any smooth function.

Equation (1.2) leads us to consider the following dynamics, which should get rid of metastabilities for a well chosen $\xi$ since it "flattens" the energy landscape in the $\xi$ direction (see [11] and Lem. 2.2 below for more precise statements):

$$
\left\{\begin{array}{l}
\mathrm{d} X_{t}=-\nabla\left(V-A_{t} \circ \xi-\beta^{-1} \ln \left(|\nabla \xi|^{-2}\right)\right)\left(X_{t}\right)|\nabla \xi|^{-2}\left(X_{t}\right) \mathrm{d} t+\sqrt{2 \beta^{-1}}|\nabla \xi|^{-1}\left(X_{t}\right) \mathrm{d} W_{t}, \\
A_{t}^{\prime}(z)=\mathbb{E}\left[F\left(X_{t}\right) \mid \xi\left(X_{t}\right)=z\right] .
\end{array}\right.
$$

The second equality in (1.4) shows that if $X_{t}$ is distributed according to the canonical measure associated with the potential $V-A \circ \xi$, then the biasing force $A_{t}^{\prime}$ is actually the derivative $A^{\prime}$ of the free energy, and the first equation in (1.4) consists in a Langevin dynamics associated to the potential $V-A \circ \xi$. Consequently, the dynamics (1.4) admits a stationary point: $A_{t}^{\prime}=A^{\prime}$ and $\operatorname{Law}\left(X_{t}\right)=\left(\int_{\mathcal{D}} \mathrm{e}^{-\beta(V-A \circ \xi)} \mathrm{d} x\right)^{-1} \mathrm{e}^{-\beta(V-A \circ \xi)} \mathrm{d} x$. The diffusion term $|\nabla \xi|^{-1}\left(X_{t}\right)$ in (1.4) (and the associated modifications of the drift term) is required to obtain natural longtime convergence results, but a constant diffusion term can also be used, see [11] for more details.

If we actually have convergence to this stationary state, we have a method, that should be efficient (i.e. that should not see the metastabilities), to sample the canonical measure up to a known perturbation $\mathrm{e}^{A \circ \xi}$. 
This algorithm has thus two applications: it allows the computation of the free energy $A$, and it can be used as an adaptative importance sampling method for the canonical measure.

The long time behavior of equation (1.4) has been studied in [11], where it has been proven that for a sufficiently regular solution, one has, in some sense, an exponential convergence to the stationary state, with a rate that is better (for a well chosen $\xi$ ) than the rate of convergence to equilibrium for (1.1).

The practical difficulty in simulating (1.4) is to compute the conditional expectation, which is a highly nonlinear term. Stochastic differential equations involving conditional expectations have already been studied, in a case where the conditional expectation is computed with respect to a random initial condition $($ see $[16,18])$ or where the variable whose conditional expectation is computed is fixed (see [7]). Our situation is much more complex since both the conditioning and the conditioned variables change with time and are affected by the previous conditional expectations.

The same difficulty arises in Lagrangian stochastic models which are commonly used in the simulation of turbulent flows (see [2]). The main difference between the system studied in [2] and (1.4) is that the authors considers a Langevin dynamics with noise only on the velocity. The lack of ellipticity then leads to additional difficulties. In our setting we are able to derive a quantitative error estimate for the particle discretization while this seems more difficult for Langevin dynamics.

In this paper, we prove that existence and uniqueness hold for equation (1.4) under suitable conditions, and we study an approximation of $X_{t}$ by an interacting particle system (see Thms. 2.3 and 2.4 below).

The paper is organized as follows. In Section 2 we state our main results.

Section 3 is devoted to some uniqueness and regularity results. More precisely, we prove that the time marginals of a solution to equation (1.4) satisfy some partial differential equation. Then, under an integrability condition on the initial condition, we prove uniqueness for the solutions to this equation, so that the nonlinear term in (1.4) is reduced to a bounded drift coefficient. We thus prove pathwise uniqueness and uniqueness in distribution for the solutions of (1.4).

Section 4 is devoted to existence results. More precisely, we introduce a regularization of the dynamics (1.4) involving two parameters $\alpha$ and $\varepsilon$, which is another nonlinear stochastic differential equation whose nonlinearity is less singular. We prove that strong existence, pathwise uniqueness and uniqueness in distribution hold for this equation and then we show that the solutions to this stochastic differential equation converge to some process which satisfies $(1.4)$ in the limit $(\alpha, \varepsilon) \rightarrow(0,0)$, yielding strong existence. We also prove that this convergence holds with rate $\mathcal{O}(\alpha+\sqrt{\varepsilon})$.

In Section 5 we introduce an interacting particle system to approximate the regularized dynamics, and we prove a propagation-of-chaos result for this particle system. We also derive a rate of convergence for this propagation of chaos.

In Section 6, we illustrate the efficiency of the particle approximation of the ABF method and the rate of those convergences with some numerical examples in small dimension.

\section{Notation}

We denote by $\mathbb{T}=\mathbb{R} / \mathbb{Z}$ the one dimensional torus, and for $x \in \mathbb{R}$, we denote by $\{x\}$ the fractional part of $x$, that can be seen as a projection of $x$ on $\mathbb{T}$. In the following, we will work in two different domains $\mathcal{D}$ : $\mathbb{T} \times \mathbb{R}^{d-1}$ or $\mathbb{T}^{d}$. The case $\mathcal{D}=\mathbb{T} \times \mathbb{R}^{d-1}$ will be called the non compact case, and the case $\mathcal{D}=\mathbb{T}^{d}$ will be called the compact case. For $x \in \mathbb{R}^{d}$, depending on the case considered, we will also denote by $\{x\}$ the element of $\mathbb{T} \times \mathbb{R}^{d-1}$ (resp. $\left.\mathbb{T}^{d}\right)$ defined by $\{x\}=\left(\left\{x^{1}\right\}, x^{2}, \ldots, x^{d}\right)\left(\operatorname{resp} .\{x\}=\left(\left\{x^{1}\right\}, \ldots,\left\{x^{d}\right\}\right)\right)$.

In the following, we will call "function defined on $\mathbb{T}$ " (resp. on $\mathbb{T} \times \mathbb{R}^{d-1}$, resp. on $\mathbb{T}^{d}$ ), a $\mathbb{Z}$-periodical (resp. $\mathbb{Z}$-periodical in the first coordinate, resp. $\mathbb{Z}^{d}$-periodical) function defined on $\mathbb{R}$ (resp. on $\mathbb{R}^{d}$ ). Integrals on $\mathbb{T}$, $\mathbb{T} \times \mathbb{R}^{d-1}$ or $\mathbb{T}^{d}$ mean integrals on $[0,1),[0,1) \times \mathbb{R}^{d-1}$ or $[0,1)^{d}$.

We denote by $\mathbb{L}^{2}\left(\mathbb{T}^{d}\right)$ the space of functions on $\mathbb{T}^{d}$ whose square is integrable on $\mathbb{T}^{d}$, and by $\mathbb{H}^{1}\left(\mathbb{T}^{d}\right)$ the space of functions in $\mathbb{L}^{2}\left(\mathbb{T}^{d}\right)$ whose weak gradient is square integrable on $\mathbb{T}^{d}$. We use similar notations on $\mathbb{T} \times \mathbb{R}^{d-1}$ and $\mathbb{T}$. 
For two functions $f$ and $g$ defined on $\mathbb{T} \times \mathbb{R}^{d-1}$ or $\mathbb{T}^{d}$, we denote $f * g$ the convolution with respect to the first coordinate, that is,

$$
f * g(x)=\int_{\mathbb{T}} f\left(x^{1}-y^{1}, x^{2 \ldots d}\right) g\left(y^{1}, x^{2 \ldots d}\right) \mathrm{d} y^{1} .
$$

If $f$ is defined on $\mathbb{T}$, we also use the notation $f * g$ to denote

$$
f * g(x)=\int_{\mathbb{T}} f\left(x^{1}-y^{1}\right) g\left(y^{1}, x^{2 \ldots d}\right) \mathrm{d} y^{1} .
$$

When $f$ and $g$ are defined on $\mathcal{D}=\mathbb{T} \times \mathbb{R}^{d-1}$ or $\mathbb{T}^{d}$, the convolution in all the coordinates is denoted $f \star g$ :

$$
f \star g(x)=\int_{\mathcal{D}} f\left(x^{1}-y^{1}, x^{2 \ldots d}-y^{2 \ldots d}\right) g\left(y^{1}, y^{2 \ldots d}\right) \mathrm{d} y^{1} \mathrm{~d} y^{2 \ldots d} .
$$

In the following, we call "probability measure on $\mathbb{T}$ " (resp. on $\mathbb{T} \times \mathbb{R}^{d-1}, \mathbb{T}^{d}$ ) a nonnegative $\mathbb{Z}$-periodical (resp. $\mathbb{Z}$-periodical with respect to the first coordinate, $\mathbb{Z}^{d}$-periodical) measure $\mu$ such that $\mu([0,1))=1$ (resp. $\left.\mu\left([0,1) \times \mathbb{R}^{d-1}\right)=1, \mu\left([0,1)^{d}\right)=1\right)$.

When $\{X\}$ is a random variable taking values in $\mathbb{T}$ (resp. in $\mathbb{T} \times \mathbb{R}^{d-1}, \mathbb{T}^{d}$ ), we call "distribution of $\{X\}$ " or "law of $\{X\}$ " the probability measure $\mu$ on $\mathbb{T}$ (resp. on $\mathbb{T} \times \mathbb{R}^{d-1}, \mathbb{T}^{d}$ ) such that

$$
\mathbb{E}[f(\{X\})]=\int f(x) \mu(\mathrm{d} x) .
$$

For a given probability measure $\mu$ on $\mathbb{T} \times \mathbb{R}^{d-1}$ (resp. a probability density $u$ ) and a given bounded function $g$, we denote $\mu^{g}$ (resp. $\left.u^{g}\left(x^{1}\right) \mathrm{d} x^{1}\right)$ the marginal on $\mathbb{T}$ of the measure $g . \mu$ (resp. $\left.g(x) u(x) \mathrm{d} x\right)$. Namely:

$$
\mu^{g}(A)=\int_{A \times \mathbb{R}^{d-1}} g \mathrm{~d} \mu
$$

and

$$
u^{g}\left(x^{1}\right)=\int_{\mathbb{R}^{d-1}} g\left(x^{1}, x^{2 \ldots d}\right) u\left(x^{1}, x^{2 \ldots d}\right) \mathrm{d} x^{2 \ldots d} .
$$

In particular, $\mu^{1}$ is the first coordinate marginal of $\mu$. When we do not specify the measure in an integral, it is the Lebesgue measure.

We will need the weighted spaces

$$
\mathbb{L}^{p}(w)=\left\{\psi \in \mathbb{L}^{p}\left(\mathbb{T} \times \mathbb{R}^{d-1}\right) \text { s.t. }\|\psi\|_{\mathbb{L}^{p}(w)} \stackrel{\text { def }}{=}\left(\int_{\mathbb{T} \times \mathbb{R}^{d-1}}|\psi|^{p} w\right)^{1 / p}<\infty\right\},
$$

for $1 \leq p<\infty$, and

$$
\mathbb{H}^{1}(w)=\left\{\psi \in \mathbb{H}^{1}\left(\mathbb{T} \times \mathbb{R}^{d-1}\right) \text { s.t. }\|\psi\|_{\mathbb{H}^{1}(w)} \stackrel{\text { def }}{=}\left(\int_{{\mathbb{T} \times \mathbb{R}^{d-1}}}\left(|\psi|^{2}+|\nabla \psi|^{2}\right) w\right)^{1 / 2}<\infty\right\}
$$

with $w(x)=\left(1+\left|x^{2 \ldots d}\right|^{2}\right)^{\lambda}$, for some $\lambda>(d-1) / 2$. Notice that $w$ does not depend on the first coordinate $x^{1}$, and that there is a positive constant $K$ such that

$$
\forall x \in \mathbb{T} \times \mathbb{R}^{d-1},|\nabla w(x)| \leq 2 \lambda\left(1+\left|x^{2 \ldots d}\right|^{2}\right)^{\lambda-1} \sum_{i=2}^{d}\left|x^{i}\right| \leq K w(x) .
$$

We will use several times the following statement. 
Lemma 1.1. For a bounded function $g$, and $u \in \mathbb{L}^{2}(w)$ one has, for some constant $K$,

$$
\left\|u^{g}\right\|_{\mathbb{L}^{2}(\mathbb{T})} \leq K\|g\|_{\mathbb{L}^{\infty}\left(\mathbb{T} \times \mathbb{R}^{d-1}\right)}\|u\|_{\mathbb{L}^{2}(w)}
$$

If moreover, $g$ has bounded derivatives and $u \in \mathbb{H}^{1}(w)$, then

$$
\left\|u^{g}\right\|_{\mathbb{H}^{1}(\mathbb{T})} \leq K\|g\|_{\mathbb{W}^{1, \infty}\left(\mathbb{T} \times \mathbb{R}^{d-1}\right)}\|u\|_{\mathbb{H}^{1}(w)}
$$

The same inequalities hold with the non weighted norms in the right-hand side, for $u$ respectively in $\mathbb{L}^{2}\left(\mathbb{T}^{d}\right)$ and $\mathbb{H}^{1}\left(\mathbb{T}^{d}\right)$.

Proof. Recall that we assumed $\lambda>\frac{d-1}{2}$, so that $\frac{1}{w}$ is integrable on $\mathbb{R}^{d}: \int_{\mathbb{R}^{d}} \frac{1}{w} \mathrm{~d} x<\infty$. Consequently, we have the estimation

$$
\begin{aligned}
\left\|u^{g}\right\|_{\mathbb{L}^{2}(\mathbb{T})}^{2} & =\int_{\mathbb{T}}\left|\int_{\mathbb{R}^{d-1}} g u\right|^{2} \\
& \leq\|g\|_{\mathbb{L}^{\infty}\left(\mathbb{T} \times \mathbb{R}^{d-1}\right)}^{2} \int_{\mathbb{T}}\left(\int_{\mathbb{R}^{d-1}}|u|^{2} w \int_{\mathbb{R}^{d-1}} \frac{1}{w}\right) \\
& \leq K\|g\|_{\mathbb{L}^{\infty}\left(\mathbb{T} \times \mathbb{R}^{d-1}\right)}^{2}\|u\|_{\mathbb{L}^{2}(w)}^{2} .
\end{aligned}
$$

The proof is similar in the space $\mathbb{H}^{1}(w)$.

In the following, $K$ will denote some positive constant, whose value can change from line to line.

\section{Assumptions And Statement of the MAin RESUlts}

In this paper, we consider a particular case of equation (1.4) to simplify the argumentation: we assume $\beta=1$ (this can be realized by a change of variable), $\mathcal{D}=\mathbb{T} \times \mathbb{R}^{d-1}$ or $\mathcal{D}=\mathbb{T}^{d}$. We consider as reaction coordinate the first coordinate function $\xi: \mathcal{D} \rightarrow \mathbb{R}$ defined by $\xi(x)=\xi\left(x^{1}, x^{2}, \ldots, x^{d}\right)=x^{1}$. This should not change the theoretical results, but will simplify the proofs. The definition (1.3) of $F$ is then reduced to

$$
F=\partial_{1} V
$$

where $V$ is defined on $\mathbb{T}^{d}$ or $\mathbb{T} \times \mathbb{R}^{d-1}$.

The two settings $\mathcal{D}=\mathbb{T}^{d}$ and $\mathcal{D}=\mathbb{T} \times \mathbb{R}^{d-1}$ will be respectively called the compact and the non-compact case. Our results hold in both settings, and the proofs are mostly identical, with some slight additional difficulties in the non compact case. Thus, in those situations, we only give the proofs in the non-compact case.

With those assumptions, equation (1.4) rewrites

$$
\mathrm{d} X_{t}=\left(-\nabla V\left(X_{t}\right)+\mathbb{E}\left[\partial_{1} V\left(X_{t}\right) \mid\left\{X_{t}^{1}\right\}\right] e_{1}\right) \mathrm{d} t+\sqrt{2} \mathrm{~d} W_{t}
$$

$e_{1}$ denoting the first vector in the canonical basis of $\mathbb{R}^{d}$. We will call solution to equation $(2.1)$ a process $\left\{X_{t}\right\}$ where $X_{t}$ satisfies (2.1). The initial condition of (2.1) is a random variable denoted $X_{0}$, and is supposed to be independent of the Brownian motion $W$. We denote by $P_{0}$ the law of $\left\{X_{0}\right\}$, which is a probability measure on $\mathcal{D}$.

To ensure the integrability of $\partial_{1} V\left(X_{t}\right)$, we make the following assumption:

Assumption i. $V$ is a twice continuously differentiable function, which has bounded first and second order partial derivatives. 
Notice that Assumption i yields boundedness of the drift coefficient in (2.1). In the compact case, Assumption $\mathrm{i}$ is satisfied as soon as $V$ is a twice differentiable function.

We have to make some assumptions on the initial condition $X_{0}$. What is needed to prove our results will depend on whether we consider the compact or the non compact case. In the compact case, we consider the following assumption:

Assumption ii. The probability measure $P_{0}$ has a density $p_{0}$ lying in $\mathbb{L}^{2}\left(\mathbb{T}^{d}\right)$ and whose first coordinate marginal $p_{0}^{1}$ is bounded from below by a positive constant. (Notice that $p_{0}^{1}$ is a probability density on $\mathbb{T}$.)

In the non compact case, we will need a stronger assumption: we have to control the decay of the initial condition at infinity, so we work in the weighted space $\mathbb{L}^{2}(w)$. We will use, in addition to Assumption ii, the following one:

Assumption iii. The density $p_{0}$ of $P_{0}$ lies in both $\mathbb{L}^{1}(w)$ and $\mathbb{L}^{2}(w)$.

Notice that Assumption iii implies that $\left\{X_{0}\right\}$ has finite moments of order less than $2 \lambda$, and that Assumption i then yields a control on the corresponding moments of any solution to (2.1), uniformly in $t \in \mathbb{R}$ :

Lemma 2.1. Under Assumptions $\mathrm{i}$ and iii, on any bounded time interval $[0, T]$, the moments of order less than $2 \lambda$ of any solution $X$ of (2.1) are bounded:

$$
\sup _{0 \leq t \leq T} \mathbb{E}\left[\left|X_{t}\right|^{2 \lambda}\right]<\infty .
$$

Proof. This comes from the boundedness of the drift coefficient $b_{s}(x)=-\nabla V(x)+\mathbb{E}\left[\partial_{1} V(X) \mid X^{1}=x^{1}\right]$, which holds in regard of Assumption i. Indeed, we have $\mathbb{E}\left[\left|X_{t}\right|^{2 \lambda}\right]=\mathbb{E}\left[\left|X_{0}+\int_{0}^{t} b_{s}\left(X_{s}\right) \mathrm{d} s+\sqrt{2} W_{t}\right|^{2 \lambda}\right] \leq$ $K\left(\mathbb{E}\left[\left|X_{0}\right|^{2 \lambda}\right]+t^{2 \lambda}+t^{\lambda}\right)$, which is bounded on $[0, T]$.

According to the following fundamental lemma, the solution to (2.1) samples efficiently the coordinate reaction state space $\mathbb{T}$.

Lemma 2.2. Denote by $P_{t}$ the law of $\left\{X_{t}\right\}$, where $X_{t}$ is a solution to equation (2.1). Then, $P_{t}^{1}$ has a density $p_{t}^{1}$, such that $p^{1}$ satisfies the heat equation on $\mathbb{T}$ with initial condition $p_{0}^{1}$. Thus, $p^{1}$ is uniquely defined on $\mathbb{T} \times[0, \infty)$, and smooth on $\mathbb{T} \times(0, \infty)$.

Proof of Lemma 2.2. Let $f$ be a smooth function on $\mathbb{T}$. One has, by Itō's formula

$$
\partial_{t} \mathbb{E}\left[f\left(X_{t}^{1}\right)\right]=-\mathbb{E}\left[f^{\prime}\left(X_{t}^{1}\right) \partial_{1} V\left(X_{t}\right)\right]+\mathbb{E}\left[f^{\prime}\left(X_{t}^{1}\right) \mathbb{E}\left[\partial_{1} V\left(X_{t}\right) \mid\left\{X_{t}^{1}\right\}\right]\right]+\mathbb{E}\left[f^{\prime \prime}\left(X_{t}^{1}\right)\right] .
$$

But, $f$ being a function on $\mathbb{T}, f^{\prime}\left(X_{t}^{1}\right)$ only depends on $\left\{X_{t}^{1}\right\}$, so that the two first terms in the right hand side cancel. Then, it holds

$$
\partial_{t} \mathbb{E}\left[f\left(X_{t}^{1}\right)\right]=\mathbb{E}\left[f^{\prime \prime}\left(X_{t}^{1}\right)\right],
$$

which is exactly the heat equation in the weak sense for $t \mapsto p_{t}^{1}, p_{t}^{1}$ being the distribution of $\left\{X_{t}^{1}\right\}$. For uniqueness and regularity of this solution, see [6], Chapter XIV.

Lemma 2.2 allows us to rewrite equation (2.1) using the distribution of $\left\{X_{t}^{1}\right\}$. Indeed, since $P_{t}^{1}$ has a density, the measure given for $A \subset[0,1)$ by $P_{t}^{\partial_{1} V}(A)=\mathbb{E}\left[\partial_{1} V\left(X_{t}\right) \mathbf{1}_{A}\left(\left\{X_{t}^{1}\right\}\right)\right]$ also has a density $p_{t}^{\partial_{1} V}$. We can thus write

$$
\begin{cases}\mathrm{d} X_{t} & =\left(-\nabla V\left(X_{t}\right)+\frac{p_{t}^{\partial_{1} V}\left(X_{t}^{1}\right)}{p_{t}^{1}\left(X_{t}^{1}\right)} e_{1}\right) \mathrm{d} t+\sqrt{2} \mathrm{~d} W_{t} \\ P_{t} & =\text { distribution of }\left\{X_{t}\right\} .\end{cases}
$$

Moreover, under Assumption ii the density $p_{t}^{1}$ satisfies $0<\inf _{\mathbb{T}} p_{0}^{1} \leq p_{t}^{1}$, uniformly in time, thanks to the maximum principle. This assumption will consequently prevent the denominator in the second term of (2.2) from vanishing. 
In view of equation (2.2), a natural particle approximation of $X_{t}$ is then obtained using the Nadaraya-Watson estimator of a conditional expectation (see [19]), given, for some parameter $\eta$ and for a positive integer $N$, by the system of $N$ stochastic differential equations

$$
\mathrm{d} X_{t, n, N}^{\eta}=\left(-\nabla V\left(X_{t, n, N}^{\eta}\right)+\frac{\sum_{m=1}^{N} \varphi_{\eta}\left(X_{t, n, N}^{\eta, 1}-X_{t, m, N}^{\eta, 1}\right) \partial_{1} V\left(X_{t, m, N}^{\eta}\right)}{\sum_{m=1}^{N} \varphi_{\eta}\left(X_{t, n, N}^{\eta, 1}-X_{t, m, N}^{\eta, 1}\right)} e_{1}\right) \mathrm{d} t+\sqrt{2} \mathrm{~d} W_{t}^{n}, 1 \leq n \leq N
$$

where $\left(W_{t}^{n}\right)$ is a sequence of independent Brownian motions, and $\varphi_{\eta}$ is a smooth approximation for the Dirac measure at the origin on $\mathbb{T}$. For the initial condition, we work with the following assumption:

Assumption iv. The initial condition of equation (2.3) is $\left(X_{0, n, N}^{\eta}\right)_{0 \leq n \leq N}=\left(X_{0, n}\right)_{0 \leq n \leq N}$, where $\left(X_{0, n}\right)_{n \in \mathbb{N}}$ is a sequence of i.i.d. random variables with density $p_{0}$, and independent of the Brownian motions $\left(W_{t}^{n}\right)_{t \geq 0}$.

We also need an assumption on the shape of $\varphi_{\eta}$. The parameter $\eta=(\alpha, \varepsilon)$ will be chosen in $(0, \infty)^{2}$, and $\varphi_{\eta}$ will have the form

$$
\varphi_{\eta}(x)=\alpha+\psi_{\varepsilon}(x)
$$

where $\psi_{\varepsilon}$ is a sequence of mollifiers on $\mathbb{T}$ as $\varepsilon \rightarrow 0$. Namely, assuming $\varepsilon<1 / 2$, $\psi_{\varepsilon}$ is a smooth non-negative $\mathbb{Z}$-periodical function, such that $\psi_{\varepsilon} \equiv 0$ on $[-1 / 2,1 / 2] \backslash[-\varepsilon, \varepsilon]$ and such that

$$
\int_{-1 / 2}^{1 / 2} \psi_{\varepsilon}=1
$$

A simple way to construct such a sequence is to consider a smooth non-negative function $\psi$ defined on $\mathbb{R}$, with support in $[-1,1]$ such that $\int_{\mathbb{R}} \psi=1$, and then consider the $\mathbb{Z}$-periodization $\psi_{\varepsilon}$ of $\psi_{\varepsilon}=\frac{1}{\varepsilon} \psi(\dot{\bar{\varepsilon}})\left(\psi_{\varepsilon}\right.$ is well defined for $\varepsilon<1 / 2$ ). This example makes the following assumption natural:

Assumption v. The function $\psi_{\varepsilon}$ satisfies

$$
\left\|\psi_{\varepsilon}\right\|_{\mathbb{L}^{\infty}(\mathbb{T})} \leq \frac{K}{\varepsilon}, \text { and }\left\|\psi_{\varepsilon}^{\prime}\right\|_{\mathbb{L}^{\infty}(\mathbb{T})} \leq \frac{K}{\varepsilon^{2}}
$$

The reason for adding a positive constant $\alpha$ to the mollifier is to avoid singularities at the denominator in the right-hand side of (2.3). Notice that (2.4) yields strong existence and uniqueness for (2.3), since the drift is globally Lipschitz continuous.

We are going to prove the following two results:

Theorem 2.3 (existence and uniqueness of the solution). In both the compact and non compact cases, under Assumption i, weak existence holds for equation (2.1). If $P$ denotes the distribution of a solution, then for all $s>0$ the time marginals $P_{s}$ of $P$ admits a density $p_{s}$, such that for all $0<t<T$,

$$
p \in \mathbb{L}^{\infty}\left((t, T), \mathbb{L}^{2}(\mathcal{D})\right) \bigcap \mathbb{L}^{2}\left((t, T), \mathbb{H}^{1}(\mathcal{D})\right)
$$

Moreover, under both Assumptions i and ii for the compact case, and under Assumptions i-iii for the non compact case, strong existence, pathwise uniqueness and uniqueness in distribution also hold, and one can take $t=0$ in $(2.5)$.

Theorem 2.4 (particle approximation of the process $X_{t}$ ). Let us consider the processes $X_{t, n, N}$ defined by (2.3). Then, under Assumptions i, ii, iv, and $\mathrm{v}$ in the compact case, and the additional Assumption iii in the noncompact case, it holds that, for any positive $T$, and for $\alpha$ and $\varepsilon$ small enough,

$$
\mathbb{E}\left[\int_{0}^{T}\left\|\frac{\sum_{n=1}^{N} \partial_{1} V\left(X_{t, n, N}^{\eta}\right) \varphi_{\eta}\left(.-X_{t, n, N}^{\eta, 1}\right)}{\sum_{n=1}^{N} \varphi_{\eta}\left(.-X_{t, n, N}^{\eta, 1}\right)}-A_{t}^{\prime}\right\|_{\left.\mathbb{L}_{\infty}^{\infty} \mathbb{T}\right)} \mathrm{d} t\right]=\mathcal{O}\left(\alpha+\sqrt{\varepsilon}+\frac{1}{\sqrt{N}} \mathrm{e}^{\frac{K}{\alpha \varepsilon^{2}}}\right) .
$$


Theorem 2.3 is a consequence of Theorem 3.8 and Corollary 4.10 below, and Theorem 2.4 is a consequence of Theorems 4.11 and 5.1 below.

The convergence rate in Theorem 2.4 is certainly not optimal. Indeed it is natural that, for the error to vanish, the number $N$ of particles should go to infinity as $\varepsilon$ goes to zero, but the dependency of $N$ on $\varepsilon$ which is required for the control of the error in Theorem 2.4 to go to zero is certainly pessimistic.

\section{Notion of solution, Regularity And uniqueness Results}

In this section we consider the Fokker-Planck equation associated to the nonlinear stochastic differential equation (2.1) and prove that uniqueness holds for weak solutions of this partial differential equation. From this uniqueness result, the study of equation (2.1) can be reduced to the study of a linear stochastic differential equation. We can thus prove uniqueness for equation (2.1).

Let us derive the Fokker-Planck equation associated to equation (2.1). Let $\psi$ be a twice continuously differentiable function. Applying Itō's formula and taking the expectation, we obtain that the law $P_{t}$ of a weak solution $\left\{X_{t}\right\}$ to equation (2.1) satisfies

$$
\begin{aligned}
\int_{\mathcal{D}} \psi(x) \mathrm{d} P_{T}(x)= & \int_{\mathcal{D}} \psi(x) \mathrm{d} P_{0}(x)-\int_{0}^{T} \int_{\mathcal{D}} \nabla \psi(x) \cdot \nabla V(x) \mathrm{d} P_{t}(x) \mathrm{d} t+\int_{0}^{T} \int_{\mathcal{D}} \Delta \psi(x) \mathrm{d} P_{t}(x) \mathrm{d} t \\
& +\int_{0}^{T} \int_{\mathcal{D}} \partial_{1} \psi(x)\left(\frac{p_{t}^{\partial_{1} V}}{p_{t}^{1}}\left(x^{1}\right)\right) \mathrm{d} P_{t}(x) \mathrm{d} t,
\end{aligned}
$$

which is a weak formulation of the following partial differential equation

$$
\partial_{t} P_{t}=\operatorname{div}\left(P_{t} \nabla V+\nabla P_{t}\right)-\partial_{1}\left(P_{t} \frac{p_{t}^{\partial_{1} V}}{p_{t}^{1}}\right)
$$

with initial condition $P_{0}$. Using integration by parts, we introduce a stronger definition for solutions to (3.2) which will allow us to prove existence and uniqueness.

Definition 3.1. In the compact case, a function $u$ is said to be a solution to (3.2) if, for any positive $T$,

- $u$ belongs to $\mathbb{L}^{\infty}\left((0, T), \mathbb{L}^{2}\left(\mathbb{T}^{d}\right)\right) \bigcap \mathbb{L}^{2}\left((0, T), \mathbb{H}^{1}\left(\mathbb{T}^{d}\right)\right)$;

- for any function $\psi \in \mathbb{H}^{1}\left(\mathbb{T}^{d}\right)$, we have:

$$
\partial_{t} \int_{\mathcal{D}} u_{t} \psi=-\int_{\mathcal{D}} u_{t} \nabla V \cdot \nabla \psi-\int_{\mathcal{D}} \nabla u_{t} \cdot \nabla \psi+\int_{\mathcal{D}} u_{t} \frac{u_{t}^{\partial_{1} V}}{u_{t}^{1}} \partial_{1} \psi
$$

in the sense of distributions in time;

- $u_{0}=p_{0}$.

In the non compact case, $u$ is said to be a solution to (3.2), if, for any positive $T$,

- $u$ belongs to $\mathbb{L}^{\infty}\left((0, T), \mathbb{L}^{2}(w)\right) \bigcap \mathbb{L}^{2}\left((0, T), \mathbb{H}^{1}(w)\right)$;

- for any $\psi \in \mathbb{H}^{1}(w)$

$$
\partial_{t} \int_{\mathcal{D}} u_{t} \psi w=-\int_{\mathcal{D}} u_{t} \nabla V \cdot(w \nabla \psi+\psi \nabla w)-\int_{\mathcal{D}} \nabla u_{t} \cdot(w \nabla \psi+\psi \nabla w)+\int_{\mathcal{D}} u_{t} \frac{u_{t}^{\partial_{1} V}}{u_{t}^{1}}\left(\partial_{1} \psi\right) w
$$

holds in the sense of distributions in time;

- $u_{0}=p_{0}$. 
Notice that (3.3) is a variational formulation of (3.2) in the space $\mathbb{L}^{2}\left(\mathbb{T}^{d}\right)$ and that (3.4) is a variational formulation of (3.2) in the space $\mathbb{L}^{2}(w)$.

These conditions make sense. Indeed, in both cases, the conditions on $u$ and $\psi$ are such that the variational formulations (3.3) and (3.4) are well defined (notice that one has $|\nabla w| \leq K w$ ). Moreover, for the compact case, if $u$ lies in $\mathbb{L}^{2}\left((0, T), \mathbb{H}^{1}\left(\mathbb{T}^{d}\right)\right)$, and satisfies (3.3) then $\partial_{t} u$ lies in $\mathbb{L}^{2}\left((0, T), \mathbb{H}^{-1}\left(\mathbb{T}^{d}\right)\right)$, so that (see [13], p. 23) $u$ lies in $\mathcal{C}\left([0, T], \mathbb{L}^{2}\left(\mathbb{T}^{d}\right)\right)$, allowing us to define the value of $u$ at time $t=0$. The same argument holds for the non compact case.

\subsection{Existence of regular densities for solutions to the nonlinear equation}

In this section, we consider a solution $X$ to equation (2.1) and we denote by $P_{t}$ the law of $\left\{X_{t}\right\}$. We show that $P_{t}$ has a density $p_{t}$, and that $p$ is a solution to equation (3.2), in the sense of Definition 3.1.

Lemma 3.2. Consider both the compact and the non compact cases. Under Assumption i, for any $t \geq 0, P_{t}$ admits a density $p_{t}$ with respect to the Lebesgue measure satisfying the following mild representation

$$
p_{t}=G_{t} \star P_{0}+\int_{0}^{t} \nabla G_{t-s} \star\left(\nabla V p_{s}\right) \mathrm{d} s-\int_{0}^{t} \partial_{1} G_{t-s} \star\left(\frac{p_{s}^{\partial_{1} V}}{p_{s}^{1}} p_{s}\right) \mathrm{d} s,
$$

where $G_{t}$ is the density of $\sqrt{2}$ times the Brownian motion on $\mathcal{D}$, namely

$$
G_{t}(x)=\frac{1}{(4 \pi t)^{d / 2}} \sum_{k \in \mathbb{Z}} \mathrm{e}^{-\frac{\left|x-k e_{1}\right|^{2}}{4 t}}
$$

for the non-compact case, and

$$
G_{t}(x)=\frac{1}{(4 \pi t)^{d / 2}} \sum_{k \in \mathbb{Z}^{d}} \mathrm{e}^{-\frac{|x-k|^{2}}{4 t}}
$$

for the compact case.

Proof. Let $\chi$ be a smooth function with compact support on $\mathbb{T} \times \mathbb{R}^{d-1}$ and $T>0$. Then, for $t \in[0, T]$, the function $\psi$ defined by

$$
\psi_{s}=G_{t-s} \star \chi,
$$

is the unique smooth solution to the following problem

$$
\left\{\begin{array}{lll}
\partial_{s} \psi=-\Delta \psi & & \text { on }(0, t) \times \mathbb{T} \times \mathbb{R}^{d-1}, \\
\psi_{t}=\chi & \text { on } \mathbb{T} \times \mathbb{R}^{d-1}
\end{array}\right.
$$

Computing $\psi_{s}\left(X_{s}\right)$ by Itō's formula and using (3.6) we get

$$
\begin{aligned}
\int_{\mathbb{T} \times \mathbb{R}^{d-1}} \psi_{t} \mathrm{~d} P_{t}= & \int_{\mathbb{T} \times \mathbb{R}^{d-1}} \psi_{0} \mathrm{~d} P_{0}-\int_{0}^{t} \int_{\mathbb{T} \times \mathbb{R}^{d-1}} \Delta \psi_{s} \mathrm{~d} P_{s} \mathrm{~d} s+\int_{0}^{t} \int_{{\mathbb{T} \times \mathbb{R}^{d-1}}} \Delta \psi_{s} \mathrm{~d} P_{s} \mathrm{~d} s \\
& -\int_{0}^{t} \int_{\mathbb{T} \times \mathbb{R}^{d-1}} \nabla \psi_{s} \cdot \nabla V \mathrm{~d} P_{s} \mathrm{~d} s+\int_{0}^{t} \int_{\mathbb{T} \times \mathbb{R}^{d-1}} \partial_{1} \psi_{s} \frac{p_{s}^{\partial_{1} V}}{p_{s}^{1}} \mathrm{~d} P_{s} \mathrm{~d} s \\
= & \int_{\mathbb{T} \times \mathbb{R}^{d-1}} \psi_{0} \mathrm{~d} P_{0}-\int_{0}^{t} \int_{\mathbb{T} \times \mathbb{R}^{d-1}} \nabla \psi_{s} \cdot \nabla V \mathrm{~d} P_{s} \mathrm{~d} s+\int_{0}^{t} \int_{\mathbb{T} \times \mathbb{R}^{d-1}} \partial_{1} \psi_{s} \frac{p_{s}^{\partial_{1} V}}{p_{s}^{1}} \mathrm{~d} P_{s} \mathrm{~d} s
\end{aligned}
$$


Using the expression of $\psi_{t}$ and Fubini's Theorem, we have:

$$
\begin{aligned}
& \int_{{\mathbb{T} \times \mathbb{R}^{d-1}}} \chi \mathrm{d} P_{t}=\int_{\mathbb{T} \times \mathbb{R}^{d-1}} \chi\left(G_{t} \star P_{0}\right)+\int_{\mathbb{T} \times \mathbb{R}^{d-1}} \chi \int_{0}^{t} \nabla G_{t-s} \star\left(P_{s} \nabla V\right) \mathrm{d} s \\
& -\int_{\mathbb{T} \times \mathbb{R}^{d-1}} \chi \int_{0}^{t} \partial_{1} G_{t-s} \star\left(\frac{p_{s}^{\partial_{1} V}}{p_{s}^{1}} P_{s}\right) \mathrm{d} s .
\end{aligned}
$$

This last equation being true for any smooth function $\chi$ with compact support, then $P_{t}$ is given by the right-hand side of (3.5), which is an integrable function, so that for any positive $t, P_{t}$ has a density $p_{t}$ satisfying (3.5).

In regard of the following lemma, $p$ necessarily satisfies some integrability conditions.

Lemma 3.3. In both the compact and the non compact case, under Assumptions i and ii, $p$ lies in $\mathbb{L}^{\infty}((0, T)$, $\left.\mathbb{L}^{2}(\mathcal{D})\right)$ for any $T>0$, and we have $\|p\|_{\mathbb{L}^{\infty}\left((0, T), \mathbb{L}^{2}(\mathcal{D})\right)} \leq C$, where $C$ is some constant only depending on $P_{0}$, $\nabla V$ and $T$.

In the non compact case, under Assumptions $\mathrm{i}$-iii, $p$ lies in $\mathbb{L}^{\infty}\left((0, T), \mathbb{L}^{2}(w)\right)$ for any $T>0$, and we have a bound $\|p\|_{\mathbb{L}^{\infty}\left((0, T), \mathbb{L}^{2}(w)\right)} \leq C$, where $C$ is some constant only depending on $P_{0}, \nabla V$ and $T$.

We only give the proof of Lemma 3.3 in the non compact case, the one in the compact case being similar.

Proof. The mild formulation (3.5) will allow us to prove that $u \in \mathbb{L}^{\infty}\left((0, T), \mathbb{L}^{2}(w)\right)$. Since $p_{0}$ lies in both $\mathbb{L}^{1}(w)$ and $\mathbb{L}^{2}(w)$, it lies in $\mathbb{L}^{q}(w)$, for any $1 \leq q \leq 2$. We first prove that we have a uniform in time estimate in $\mathbb{L}^{q}(w)$, $1 \leq q \leq 2$, for $p_{t}$.

From equation (3.5), it follows

$$
\left\|p_{t}\right\|_{\mathbb{L}^{q}(w)} \leq\left\|p_{0}\right\|_{\mathbb{L}^{q}(w)}+\int_{0}^{t}\left\|\nabla G_{t-s} \star\left(\nabla V p_{s}\right)\right\|_{\mathbb{L}^{q}(w)}+\left\|\partial_{1} G_{t-s} \star\left(\frac{p_{s}^{\partial_{1} V}}{p_{s}^{1}} p_{s}\right)\right\|_{\mathbb{L}^{q}(w)} \mathrm{d} s .
$$

It holds, from Jensen's inequality,

$$
\begin{aligned}
\left\|\nabla G_{t-s} \star\left(\nabla V p_{s}\right)\right\|_{\mathbb{L}^{q}(w)}^{q} & \leq K \int_{\mathbb{T} \times \mathbb{R}^{d-1}}\left(\left|\nabla G_{t-s}\right| \star p_{s}\right)^{q} w \leq K \int_{{\mathbb{T} \times \mathbb{R}^{d-1}}}\left(\left|\nabla G_{t-s}\right|^{q} \star p_{s}\right) w \\
& =K \int_{{\mathbb{T} \times \mathbb{R}^{d-1}}} \int_{\mathbb{T} \times \mathbb{R}^{d-1}}\left|\nabla G_{t-s}(y)\right|^{q} p_{s}(x-y) w(x) \mathrm{d} x \mathrm{~d} y .
\end{aligned}
$$

Now, notice that $w(x) \leq K\left(1+\left|y^{2 \ldots d}\right|^{2 \lambda}\right) w(x-y) \stackrel{\text { def }}{=} \pi(y) w(x-y)$, so that

$$
\begin{aligned}
\left\|\nabla G_{t-s} \star\left(\nabla V p_{s}\right)\right\|_{\mathbb{L}^{q}(w)}^{q} & \leq K \int_{\mathbb{T} \times \mathbb{R}^{d-1}} \int_{\mathbb{T} \times \mathbb{R}^{d-1}}\left|\nabla G_{t-s}(y)\right|^{q} \pi(y) p_{s}(x-y) w(x-y) \mathrm{d} x \mathrm{~d} y \\
& =K\left\|p_{s}\right\|_{\mathbb{L}^{1}(w)} \int_{\mathbb{T} \times \mathbb{R}^{d-1}}\left|\nabla G_{t-s}(y)\right|^{q} \pi(y) \mathrm{d} y .
\end{aligned}
$$

In view of Lemma 2.1, $\left\|p_{s}\right\|_{\mathbb{L}^{1}(w)}$ is bounded. Moreover, one has for $0 \leq s \leq t \leq T$,

$$
\begin{aligned}
\left|\nabla G_{t-s}(y)\right|^{q} \pi(y) & =\left|(4 \pi(t-s))^{-d / 2} \sum_{k \in \mathbb{Z}}-\frac{y-k e_{1}}{2(t-s)} \mathrm{e}^{-\frac{\left|y-k e_{1}\right|}{4(t-s)}}\right|^{q}\left(1+\left|y^{2 \ldots d}\right|^{2 \lambda}\right) \\
& \leq \frac{K}{(t-s)^{q(d+1) / 2}}\left|\left(1+\frac{\left|y^{2 \ldots d}\right|^{2 \lambda / q}}{(t-s)^{\lambda / q}}\right) \sum_{k \in \mathbb{Z}} \frac{\left|y-k e_{1}\right|}{\sqrt{t-s}} \mathrm{e}^{-\frac{\left|y-k e_{1}\right|^{2}}{4(t-s)}}\right|^{q} .
\end{aligned}
$$


Then, since a function $f$ with polynomial growth satisfies $f(x) \mathrm{e}^{-x^{2}} \leq K \mathrm{e}^{-x^{2} / 2}$ for some constant $K$, using Hölder's inequality, we deduce,

$$
\begin{aligned}
& \left|\nabla G_{t-s}(y)\right|^{q} \pi(y) \leq \frac{K}{(t-s)^{q(d+1) / 2}}\left|\sum_{k \in \mathbb{Z}} \mathrm{e}^{-\frac{\left|y-k e_{1}\right|^{2}}{8(t-s)}}\right|^{q} \\
& \leq \frac{K}{(t-s)^{q(d+1) / 2}}\left|\sum_{k \in \mathbb{Z}} \mathrm{e}^{-\frac{q\left|y-k e_{1}\right|^{2}}{16(t-s)}}\right|\left|\sum_{k \in \mathbb{Z}} \mathrm{e}^{-\frac{q^{\prime}\left|y-k e_{1}\right|^{2}}{16 T}}\right|^{\frac{q}{q^{\prime}}}
\end{aligned}
$$

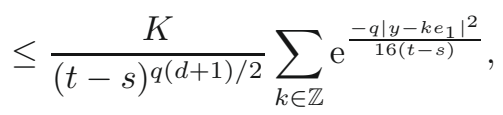

where $q^{\prime}$ satisfies $\frac{1}{q}+\frac{1}{q^{\prime}}=1$. Consequently, it holds, for $0 \leq s \leq t \leq T$,

$$
\left(\int_{\mathbb{T} \times \mathbb{R}^{d-1}}\left|\nabla G_{t-s}(y)\right|^{q} \pi(y) \mathrm{d} y\right)^{1 / q} \leq \frac{K}{(t-s)^{(d+1) / 2-d / 2 q}}
$$

The last term in (3.7) can be bounded in the same way, so we deduce that $\int_{0}^{t}\left\|\nabla G_{t-s} \star\left(\nabla V p_{s}\right)\right\|_{\mathbb{L}^{q}(w)}+$ $\left\|\partial_{1} G_{t-s} \star\left(\frac{p_{s}^{\partial_{1} V}}{p_{s}^{1}} p_{s}\right)\right\|_{\mathbb{L}^{q}(w)} \mathrm{d} s$ is finite as soon as

$$
1 \leq q<\frac{d}{d-1}
$$

In view of (3.7), $p$ lies in $\mathbb{L}^{\infty}\left((0, T), \mathbb{L}^{q}\left(\mathbb{T} \times \mathbb{R}^{d-1}\right)\right)$ for all $T$ and all $q$ satisfying (3.9), and we have a bound on its norm depending only on $P_{0}, \nabla V$ and $T$. We now bootstrap this estimate to reach a uniform-in-time $\mathbb{L}^{2}(w)$ bound for $p$.

Let $n_{0}$ be an integer large enough so that

$$
\frac{n_{0}+1}{n_{0}+1 / 2}<\frac{d}{d-1}
$$

and define for $n=0, \ldots, n_{0}, q=\frac{n_{0}+1}{n_{0}+1 / 2}$ and $q_{n}=\left(\frac{1}{q}+n\left(\frac{1}{q}-1\right)\right)^{-1}$. Notice that $\left(q_{n}\right)_{n=0 \ldots n_{0}}$ satisfies $q_{0}=q$, $q_{n_{0}}=2$ and

$$
1+\frac{1}{q_{n+1}}=\frac{1}{q_{n}}+\frac{1}{q}
$$

so that, according to Young's Inequality, convolution continuously maps $\mathbb{L}^{q_{n}} \times \mathbb{L}^{q}$ to $\mathbb{L}^{q_{n+1}}$. Consequently, we have for $n<n_{0}$

$$
\begin{aligned}
\left\|\nabla G_{t-s} \star\left(\nabla V p_{s}\right)\right\|_{\mathbb{L}^{q_{n+1}}(w)} & \leq K\left(\int_{\mathbb{T} \times \mathbb{R}^{d-1}}\left(\left|\nabla G_{t-s}\right| \star p_{s}\right)^{q_{n+1}}(x) w(x) \mathrm{d} x\right)^{1 / q_{n+1}} \\
& =K\left(\int_{\mathbb{T} \times \mathbb{R}^{d-1}}\left(\int_{\mathbb{T} \times \mathbb{R}^{d-1}}\left|\nabla G_{t-s}\right|(y) p_{s}(x-y) \mathrm{d} y\right)^{q_{n+1}} w(x) \mathrm{d} x\right)^{1 / q_{n+1}} .
\end{aligned}
$$


We have $w(x) \leq w(x-y) \pi(y) \leq w(x-y)^{q_{n+1} / q_{n}} \pi(y)$, since $q_{n} \leq q_{n+1}$, yielding, by Young's inequality and the polynomial growth of $\pi$,

$$
\begin{aligned}
\left\|\nabla G_{t-s} \star\left(\nabla V p_{s}\right)\right\|_{\mathbb{L}^{q_{n+1}}(w) \leq} & K\left(\int _ { \mathbb { T } \times \mathbb { R } ^ { d - 1 } } \left(\int_{{\mathbb{T} \times \mathbb{R}^{d-1}}\left|\nabla G_{t-s}\right|(y) \pi(y)^{1 / q_{n+1}}}\right.\right. \\
& \left.\left.\times p_{s}(x-y) w(x-y)^{1 / q_{n}} \mathrm{~d} y\right)^{q_{n+1}} \mathrm{~d} x\right)^{1 / q_{n+1}} \\
= & K\left\|\left(\left|\nabla G_{t-s}\right| \pi^{1 / q_{n+1}}\right) \star\left(p_{s} w^{1 / q_{n}}\right)\right\|_{\mathbb{L}^{q_{n+1}}\left(\mathbb{T} \times \mathbb{R}^{d-1}\right)} \\
\leq & K\left\|\left|\nabla G_{t-s}\right| \pi^{1 / q_{n+1}}\right\|_{\mathbb{L}^{q}\left(\mathbb{T} \times \mathbb{R}^{d-1}\right)}\left\|p_{s}\right\|_{\mathbb{L}^{q_{n}}(w)} \\
\leq & \frac{K}{(t-s)^{(d+1) / 2-d /(2 q)}}\left\|p_{s}\right\|_{\mathbb{L}^{q_{n}}(w)},
\end{aligned}
$$

the last inequality being proved in the same way as (3.8) is. As a result, for $n<n_{0}$,

$$
\left\|p_{t}\right\|_{\mathbb{L}^{q_{n+1}(w)}} \leq\left\|p_{0}\right\|_{\mathbb{L}^{q_{n+1}(w)}}+K \int_{0}^{t} \frac{\left\|p_{s}\right\|_{\mathbb{L}^{q_{n}(w)}}}{(t-s)^{(d+1) / 2-d /(2 q)}} \mathrm{d} s .
$$

By induction on $n$, since $\frac{1}{(t-s)^{(d+1) / 2-d /(2 q)}}$ is integrable on $[0, t]$, this estimate shows that $p$ lies in $\mathbb{L}^{\infty}((0, T)$, $\mathbb{L}^{2}(w)$ ), for all positive $T$. Since we control $\sup _{t \in[0, T]}\left\|p_{t}\right\|_{\mathbb{L}^{q_{0}}(w)}$ by a constant depending only on $P_{0}, \nabla V$ and $T$, we also have such a control on $\sup _{t \in[0, T]}\left\|p_{t}\right\|_{\mathbb{L}^{2}(w)}$.

Now, we prove that $p$ is a solution to equation (3.2) in the sense of Definition 3.1. First, we show that it satisfies the regularity condition.

Lemma 3.4. In the compact case, under Assumptions i and ii, one has

$$
p \in \mathbb{L}^{\infty}\left((0, T), \mathbb{L}^{2}\left(\mathbb{T}^{d}\right)\right) \bigcap \mathbb{L}^{2}\left((0, T), \mathbb{H}^{1}\left(\mathbb{T}^{d}\right)\right) .
$$

Moreover $\|p\|_{\mathbb{L}^{\infty}\left((0, T), \mathbb{L}^{2}\left(\mathbb{T}^{d}\right)\right)}+\|p\|_{\mathbb{L}^{2}\left((0, T), \mathbb{H}^{1}\left(\mathbb{T}^{d}\right)\right)} \leq K$, where $K$ only depends on $\nabla V, P_{0}$ and $T$.

In the non compact case, with the additional Assumption iii, one has

$$
p \in \mathbb{L}^{\infty}\left((0, T), \mathbb{L}^{2}(w)\right) \bigcap \mathbb{L}^{2}\left((0, T), \mathbb{H}^{1}(w)\right) .
$$

Moreover $\|p\|_{\mathbb{L}^{\infty}\left((0, T), \mathbb{L}^{2}(w)\right)}+\|p\|_{\mathbb{L}^{2}\left((0, T), \mathbb{H}^{1}(w)\right)} \leq K$, where $K$ only depends on $\nabla V, P_{0}$ and $T$.

Proof. According to Assumption ii, $p_{0}$ lies in $\mathbb{L}^{2}(\mathcal{D})$. Consequently, from Lemma 3.3, we know that $P_{t}$ has a density $p_{t}$ such that $p \in \mathbb{L}^{\infty}\left((0, T), \mathbb{L}^{2}(\mathcal{D})\right)$. We now prove that $p$ lies in $\mathbb{L}^{2}\left((0, T), \mathbb{H}^{1}(\mathcal{D})\right)$. We know that $p$ lies in $\mathbb{L}^{\infty}\left((0, T), \mathbb{L}^{2}(\mathcal{D})\right) \subset \mathbb{L}^{2}\left((0, T), \mathbb{L}^{2}(\mathcal{D})\right)$, and that $\frac{p^{\partial_{1} V}}{p^{1}}$ is in $\mathbb{L}^{\infty}([0, T] \times \mathcal{D})$, so that the function $f$ defined by

$$
f=\operatorname{div}(p \nabla V)-\partial_{1}\left(\frac{p^{\partial_{1} V}}{p^{1}} p\right)
$$

lies in $\mathbb{L}^{2}\left((0, T), \mathbb{H}^{-1}(\mathcal{D})\right)$. Consequently, it can be shown, for example using a Galerkin approximation (see [6], Chap. XVIII) that the problem

$$
\left\{\begin{aligned}
\partial_{t} v-\Delta v & =f \\
v_{0} & =p_{0}
\end{aligned}\right.
$$

admits a unique weak solution $v$ in the space $\mathbb{L}^{\infty}\left((0, T), \mathbb{L}^{2}(\mathcal{D})\right) \cap \mathbb{L}^{2}\left((0, T), \mathbb{H}^{1}(\mathcal{D})\right)$. Here, "weak solution" means that for any $\psi$ in $\mathbb{H}^{1}(\mathcal{D})$,

$$
\partial_{t} \int_{\mathcal{D}} \psi v_{t}+\int_{\mathcal{D}} \nabla \psi \nabla v_{t}=\int_{\mathcal{D}} \psi f
$$


holds. Thanks to an a priori estimate, we can find a bound $K$ depending only on $\nabla V, P_{0}$ and $T$, such that this weak solution lies in the ball of radius $C$ in the spaces $\mathbb{L}^{\infty}\left((0, T), \mathbb{L}^{2}(\mathcal{D})\right)$ and $\mathbb{L}^{2}\left((0, T), \mathbb{H}^{1}(\mathcal{D})\right)$. For the non compact case, notice that under Assumption iii, $f$ satisfies for any $\psi \in \mathbb{H}^{1}(w)$,

$$
\begin{aligned}
\left|\int_{\mathbb{T} \times \mathbb{R}^{d-1}} f \psi w\right|= & \left|\int_{\mathbb{T} \times \mathbb{R}^{d-1}} p \nabla V \cdot \nabla(\psi w)-\frac{p^{\partial_{1} V}}{p^{1}} p \partial_{1}(\psi w)\right| \\
& \leq K \int_{\mathbb{T} \times \mathbb{R}^{d-1}}|p \nabla \psi| w+K \int_{\mathbb{T} \times \mathbb{R}^{d-1}}|p \psi| w \leq K\|\psi\|_{\mathbb{H}^{1}(w)},
\end{aligned}
$$

the last bound being deduced from Lemma 3.3. From the following a priori estimate,

$$
\begin{aligned}
\frac{1}{2} \partial_{t}\left\|v_{t}\right\|_{\mathbb{L}^{2}(w)}^{2} & =-\int_{\mathbb{T} \times \mathbb{R}^{d-1}} \nabla v_{t} \nabla\left(w v_{t}\right)+\int_{\mathbb{T} \times \mathbb{R}^{d-1}} f v_{t} w \\
& \leq-\int_{\mathbb{T} \times \mathbb{R}^{d-1}}\left|\nabla v_{t}\right|^{2} w+K \int_{{\mathbb{T} \times \mathbb{R}^{d-1}}\left|v_{t} \nabla v_{t}\right| w+K\left\|v_{t}\right\|_{\mathbb{H}^{1}(w)}} \\
& \leq-\frac{1}{2}\left\|\nabla v_{t}\right\|_{\mathbb{L}^{2}(w)}^{2}+K\left\|v_{t}\right\|_{\mathbb{L}^{2}(w)}^{2}+K
\end{aligned}
$$

standard arguments show that $v$ also lies in $\mathbb{L}^{\infty}\left((0, T), \mathbb{L}^{2}(w)\right) \bigcap \mathbb{L}^{2}\left((0, T), \mathbb{H}^{1}(w)\right)$, if $p_{0} \in \mathbb{L}^{2}(w)$.

We are now going to show that $v$ is actually equal to the function $p$. For a fixed $t$ in $[0, T]$, consider $\psi_{s}=G_{t-s} \star \chi$, solution to the problem (3.6), where $\chi$ is some test function, and compute $\partial_{s} \int_{\mathcal{D}} \psi_{s} v_{s}$. From [17], p. 261, Lemma 1.2, we obtain

$$
\partial_{s} \int_{\mathcal{D}} \psi_{s} v_{s}=\int_{\mathcal{D}} \psi_{s} f
$$

in the sense of distributions. Using the expression of $\psi_{s}$, this equation rewrites

$$
\partial_{s} \int_{\mathcal{D}}\left(G_{t-s} \star \chi\right) v_{s}=\int_{\mathcal{D}}\left(G_{t-s} \star \chi\right) f
$$

which is equivalent to

$$
\partial_{s} \int_{\mathcal{D}} \chi\left(G_{t-s} \star v_{s}\right)=\int_{\mathcal{D}} \chi\left(G_{t-s} \star f\right) .
$$

Since $v \in \mathbb{L}^{2}\left((0, T), \mathbb{H}^{1}(\mathcal{D})\right)$, and $\partial_{s} v \in \mathbb{L}^{2}\left((0, T), \mathbb{H}^{-1}(\mathcal{D})\right)$, then $v$ lies in $\mathcal{C}\left((0, T), \mathbb{L}^{2}(\mathcal{D})\right)$ (see [6], Chap. XVIII, Sect. 1, Thm. 1), so that the left hand side in (3.14) is the derivative with respect of $s$ of a function which is continuous in $s$. Moreover, one has

$$
G_{t-s} \star f=\nabla G_{t-s} \star(p \nabla V)-\partial_{1} G_{t-s} \star\left(\frac{p^{\partial_{1} V}}{p^{1}} p\right) \in \mathbb{L}^{1}\left((0, t), \mathbb{L}^{2}(\mathcal{D})\right),
$$

so that the right hand side in (3.14) is integrable in time. Consequently, integrating on $[0, t]$, one finds

$$
\int_{\mathcal{D}} \chi v_{t}=\int_{\mathcal{D}} \chi\left(G_{t} \star p_{0}\right)+\int_{\mathcal{D}} \int_{0}^{t} \chi\left(\nabla G_{t-s} \star\left(\nabla V p_{s}\right)\right) \mathrm{d} s-\int_{\mathcal{D}} \int_{0}^{t} \chi\left(\partial_{1} G_{t-s} \star\left(\frac{p_{s}^{\partial_{1} V}}{p_{s}^{1}} p_{s}\right)\right) \mathrm{d} s .
$$

Identifying in the sense of distribution, one has

$$
v_{t}=G_{t} \star p_{0}+\int_{0}^{t} \nabla G_{t-s} \star\left(\nabla V p_{s}\right) \mathrm{d} s-\int_{0}^{t} \partial_{1} G_{t-s} \star\left(\frac{p_{s}^{\partial_{1} V}}{p_{s}^{1}} p_{s}\right) \mathrm{d} s .
$$

The right hand side in (3.15) is exactly the right hand side in (3.5), and (3.15) holds for all $t>0$, so that $v=p$, and the regularity we wanted on $p$ actually holds. 
We finish this section by proving:

Lemma 3.5. The function p satisfies equation (3.2) in the sense of Definition 3.1.

Proof. According to Lemma 3.4, in the compact case (resp. in the non compact case), for any $t>0, p$ lies in $\mathbb{L}^{\infty}\left((0, T), \mathbb{L}^{2}\left(\mathbb{T}^{d}\right) \bigcap \mathbb{L}^{2}\left((0, T), \mathbb{H}^{1}\left(\mathbb{T}^{d}\right)\right)\right.$ (resp. in $\mathbb{L}^{\infty}\left((0, T), \mathbb{L}^{2}(w) \bigcap \mathbb{L}^{2}\left((0, T), \mathbb{H}^{1}(w)\right)\right)$. Moreover, thanks to Itō's Formula, $p$ satisfies equation (3.1) for any smooth test function $\psi$. But, according to the regularity of $p_{t}$, and by the density of smooth functions in $\mathbb{H}^{1}\left(\mathbb{T}^{d}\right)$ (resp. in $\mathbb{H}^{1}(w)$ ), equation (3.3) holds for any $\psi$ in $\mathbb{H}^{1}\left(\mathbb{T}^{d}\right.$ ) (resp. (3.4) holds for any $\psi$ in $\mathbb{H}^{1}(w)$ ). This means that $p_{t}$ is a solution to (3.2) in the sense of Definition 3.1.

\subsection{Uniqueness results}

In this section we prove that uniqueness holds for solutions of equation (3.2) in the sense of Definition 3.1, yielding uniqueness for solutions of the nonlinear equation (2.1).

\subsubsection{Uniqueness for the Fokker-Planck equation}

Theorem 3.6. In the compact case, under Assumptions i and ii or in the non compact case under Assumptions i-iii, uniqueness holds for the solutions to the Fokker-Planck equation (3.2) in the sense of Definition 3.1.

Proof. We only give the proof in the non compact case, which can be adapted straightforwardly for the compact case by performing the same computations in the space $\mathbb{L}^{2}\left(\mathbb{T}^{d}\right)$. Let $u$ and $v$ be two solutions of (3.2) in the sense of Definition 3.1 with same initial condition $u_{0}=v_{0}$. We use Grönwall's Lemma to prove that $\left\|u_{t}-v_{t}\right\|_{\mathbb{L}^{2}(w)}=0$ for all $t>0$. Adapting the proof of [17], p. 261, Lemma 1.2, one has $\frac{1}{2} \partial_{t}\left\|u_{t}-v_{t}\right\|_{\mathbb{L}^{2}(w)}^{2}=$ $\int_{\mathbb{T} \times \mathbb{R}^{d-1}}\left(u_{t}-v_{t}\right) \partial_{t}\left(u_{t}-v_{t}\right) w$. Consequently, since $u$ and $v$ satisfy Definition 3.1, and using (1.5) and Assumption i, it holds that

$$
\begin{aligned}
\frac{1}{2} \partial_{t}\left\|u_{t}-v_{t}\right\|_{\mathbb{L}^{2}(w)}^{2} \leq & K\left\|u_{t}-v_{t}\right\|_{\mathbb{L}^{2}(w)}^{2}+K\left\|u_{t}-v_{t}\right\|_{\mathbb{L}^{2}(w)}\left\|\nabla u_{t}-\nabla v_{t}\right\|_{\mathbb{L}^{2}(w)}-\left\|\nabla u_{t}-\nabla v_{t}\right\|_{\mathbb{L}^{2}(w)}^{2} \\
& +\int_{\mathbb{T} \times \mathbb{R}^{d-1}} \partial_{1}\left(u_{t}-v_{t}\right)\left(u_{t} \frac{u_{t}^{\partial_{1} V}}{u_{t}^{1}}-v_{t} \frac{v_{t}^{\partial_{1} V}}{v_{t}^{1}}\right) w .
\end{aligned}
$$

We want to estimate the last term. Notice that, thanks to Lemma $2.2, u^{1}=v^{1}$, so that

$$
\begin{aligned}
\int_{\mathbb{T} \times \mathbb{R}^{d-1}} \partial_{1}\left(u_{t}-v_{t}\right)\left(u_{t} \frac{u_{t}^{\partial_{1} V}}{u_{t}^{1}}-v_{t} \frac{v_{t}^{\partial_{1} V}}{v_{t}^{1}}\right) w= & \int_{\mathbb{T} \times \mathbb{R}^{d-1}} \partial_{1}\left(u_{t}-v_{t}\right) u_{t} \frac{u_{t}^{\partial_{1} V}-v_{t}^{\partial_{1} V}}{u_{t}^{1}} w \\
& +\int_{\mathbb{T}^{1} \times \mathbb{R}^{d-1}} \partial_{1}\left(u_{t}-v_{t}\right)\left(u_{t}-v_{t}\right) \frac{v_{t}^{\partial_{1} V}}{u_{t}^{1}} w
\end{aligned}
$$

Since $\partial_{1} V$ is bounded, the second term in the right-hand side is smaller than

$$
K\left\|u_{t}-v_{t}\right\|_{\mathbb{L}^{2}(w)}\left\|\nabla u_{t}-\nabla v_{t}\right\|_{\mathbb{L}^{2}(w)}
$$

and the first term is smaller than

$$
\left\|\nabla u_{t}-\nabla v_{t}\right\|_{\mathbb{L}^{2}(w)}\left(\int_{\mathbb{T} \times \mathbb{R}^{d-1}}\left(\frac{u_{t}}{u_{t}^{1}}\right)^{2}\left(u_{t}^{\partial_{1} V}-v_{t}^{\partial_{1} V}\right)^{2} w\right)^{1 / 2}
$$


Then,

$$
\begin{aligned}
\left(\int_{\mathbb{T} \times \mathbb{R}^{d-1}}\left(\frac{u_{t}}{u_{t}^{1}}\right)^{2}\left(u_{t}^{\partial_{1} V}-v_{t}^{\partial_{1} V}\right)^{2} w\right)^{1 / 2} & =\left(\int_{\mathbb{T}}\left(\frac{u_{t}^{\partial_{1} V}-v_{t}^{\partial_{1} V}}{u_{t}^{1}}\right)^{2}\left(\int_{\mathbb{R}^{d-1}}\left(u_{t}\right)^{2} w\right)\right)^{1 / 2} \\
& \leq\left\|\frac{1}{u_{t}^{1}}\left(u_{t}^{\partial_{1} V}-v_{t}^{\partial_{1} V}\right)\right\|_{\mathbb{L}^{\infty}(\mathbb{T})}\left\|u_{t}\right\|_{\mathbb{L}^{2}(w)} .
\end{aligned}
$$

The function $t \mapsto\left\|u_{t}\right\|_{\mathbb{L}^{2}(w)}$ is bounded on $[0, T]$, and, thanks to Lemma 2.2, Assumption ii and the maximum principle, $u^{1}$ is bounded from below by some positive constant, so that

$$
\left(\int_{\mathbb{T} \times \mathbb{R}^{d-1}}\left(\frac{u_{t}}{u_{t}^{1}}\right)^{2}\left(u_{t}^{\partial_{1} V}-v_{t}^{\partial_{1} V}\right)^{2} w\right)^{1 / 2} \leq K\left\|u_{t}^{\partial_{1} V}-v_{t}^{\partial_{1} V}\right\|_{\mathbb{L}^{\infty}(\mathbb{T})} .
$$

To conclude, notice that, for any positive $\gamma, \mathbb{H}^{1 / 2+\gamma}(\mathbb{T})$ continuously imbeds in $\mathcal{C}(\mathbb{T})$ (see [1], p. 217). Consequently, interpolating $\mathbb{H}^{1}(\mathbb{T})$ and $\mathbb{L}^{2}(\mathbb{T})$ (see [13], p. 49), we obtain for a function $f$ in $\mathbb{H}^{1}(\mathbb{T})$ and $\gamma \in\left(0, \frac{1}{2}\right)$,

$$
\|f\|_{\mathbb{L}^{\infty}(\mathbb{T})} \leq K\|f\|_{\mathbb{H}^{1 / 2+\gamma}(\mathbb{T})} \leq K\|f\|_{\mathbb{L}^{2}(\mathbb{T})}^{1 / 2-\gamma}\|f\|_{\mathbb{H}^{1}(\mathbb{T})}^{1 / 2+\gamma}
$$

All the previous inequalities give us

$$
\begin{aligned}
\frac{1}{2} \partial_{t}\left\|u_{t}-v_{t}\right\|_{\mathbb{L}^{2}(w)}^{2}+\left\|\nabla u_{t}-\nabla v_{t}\right\|_{\mathbb{L}^{2}(w)}^{2} \leq & K\left\|u_{t}-v_{t}\right\|_{\mathbb{L}^{2}(w)}\left\|\nabla u_{t}-\nabla v_{t}\right\|_{\mathbb{L}^{2}(w)}+K\left\|u_{t}-v_{t}\right\|_{\mathbb{L}^{2}(w)}^{2} \\
& +K\left\|u_{t}^{\partial_{1} V}-v_{t}^{\partial_{1} V}\right\|_{\mathbb{L}^{2}(\mathbb{T})}^{1 / 2-\gamma}\left\|u_{t}^{\partial_{1} V}-v_{t}^{\partial_{1} V}\right\|_{\mathbb{H}^{1}(\mathbb{T})}^{1 / 2+\gamma}\left\|\nabla u_{t}-\nabla v_{t}\right\|_{\mathbb{L}^{2}(w)} .
\end{aligned}
$$

We finally obtain, from Lemma 1.1 and Young's inequality $a b \leq \varepsilon a^{p}+q^{-1}(p \varepsilon)^{-q / p} b^{q}$, holding true for any positive $a, b, \varepsilon, p$ and $q$ such that $\frac{1}{p}+\frac{1}{q}=1$,

$$
\partial_{t}\left\|u_{t}-v_{t}\right\|_{\mathbb{L}^{2}(w)}^{2}+\left\|\nabla u_{t}-\nabla v_{t}\right\|_{\mathbb{L}^{2}(w)}^{2} \leq K\left\|u_{t}-v_{t}\right\|_{\mathbb{L}^{2}(w)}^{2},
$$

yielding uniqueness through Grönwall's Lemma.

Remark 3.7. A more natural uniqueness proof can be performed, using an entropy estimate. In particular, this proof does not require the introduction of the weighted spaces. Unfortunately, it does not apply to the solutions in the sense of Definition 3.1. Uniqueness actually holds in the subspace of functions such that the following computations make sense.

Let $u$ and $v$ be two solutions of (3.2) with same initial condition $u_{0}=v_{0}$. Notice that from Lemma 2.2, the functions $u^{1}$ and $v^{1}$ are equal. Define the relative entropy of $u$ with respect to $v$ :

$$
E(t)=\int_{\mathbb{T} \times \mathbb{R}^{d-1}} u \log \frac{u}{v}
$$


If all quantities involved are finite, it holds that

$$
\begin{aligned}
& E^{\prime}(t)=\partial_{t}\left(\int_{\mathbb{T} \times \mathbb{R}^{d-1}} u\right)+\int_{\mathbb{T} \times \mathbb{R}^{d-1}} \partial_{t} u \log \frac{u}{v}-\int_{\mathbb{T} \times \mathbb{R}^{d-1}} \partial_{t} v \frac{u}{v} \\
& =0-\int_{\mathbb{T} \times \mathbb{R}^{d-1}} u \nabla V \nabla \log \frac{u}{v}-\int_{\mathbb{T} \times \mathbb{R}^{d-1}} \nabla u \nabla \log \frac{u}{v}+\int_{\mathbb{T} \times \mathbb{R}^{d-1}} u \frac{u^{\partial_{1} V}}{u^{1}} \partial_{1} \log \frac{u}{v} \\
& +\int_{\mathbb{T} \times \mathbb{R}^{d-1}} v \nabla V \nabla \frac{u}{v}+\int_{\mathbb{T} \times \mathbb{R}^{d-1}} \nabla v \nabla \frac{u}{v}-\int_{\mathbb{T} \times \mathbb{R}^{d-1}} v \frac{v^{\partial_{1} V}}{u^{1}} \partial_{1} \frac{u}{v} \\
& =-\int_{\mathbb{T} \times \mathbb{R}^{d-1}} \frac{v^{2}}{u}\left|\nabla \frac{u}{v}\right|^{2}+\int_{\mathbb{T} \times \mathbb{R}^{d-1}}\left(u^{\partial_{1} V}-v^{\partial_{1} V}\right) \frac{v}{u^{1}} \partial_{1} \frac{u}{v} .
\end{aligned}
$$

But, using Csiszár-Kullback inequality, it holds

$$
\begin{aligned}
& \int_{\mathbb{T} \times \mathbb{R}^{d-1}}\left(u^{\partial_{1} V}-v^{\partial_{1} V}\right) \frac{v}{u^{1}} \partial_{1} \frac{u}{v} \leq K \int_{\mathbb{T}^{\prime} \times \mathbb{R}^{d-1}} v\left|\partial_{1} \frac{u}{v}\right|\left\|\frac{u}{u^{1}}-\frac{v}{u^{1}}\right\|_{T V\left(\mathbb{R}^{d-1}\right)} \\
& \leq K \int_{\mathbb{T} \times \mathbb{R}^{d-1}} v\left|\partial_{1} \frac{u}{v}\right|\left(\int_{\mathbb{R}^{d-1}}\left(\frac{u}{u^{1}} \log \frac{u}{v}\right)\right)^{1 / 2} .
\end{aligned}
$$

In conclusion, we find

$$
E^{\prime}(t) \leq-\int_{\mathbb{T} \times \mathbb{R}^{d-1}} \frac{v^{2}}{u}\left|\nabla \frac{u}{v}\right|^{2}+K\left(\int_{\mathbb{T} \times \mathbb{R}^{d-1}} \frac{v^{2}}{u}\left|\partial_{1} \frac{u}{v}\right|^{2}\right)^{1 / 2}(E(t))^{1 / 2} .
$$

We can conclude the proof using Young's inequality and then Grönwall's Lemma.

\subsubsection{Uniqueness for the nonlinear process}

Theorem 3.8. Pathwise uniqueness and uniqueness in law hold for equation (2.1) in the compact case under Assumptions $\mathrm{i}$ and ii, and in the non compact case under Assumptions i-iii.

Proof. As stated in Lemma 3.5, if $X$ solves (2.1), then $\left\{X_{t}\right\}$ admits a density $p_{t}$ such that $p$ satisfies (3.2) in the sense of Definition 3.1. Thus, in regard of Theorem 3.6, $p_{t}$ is uniquely defined. Consequently, equation (2.1) rewrites

$$
\mathrm{d} X_{t}=\left(-\nabla V\left(X_{t}\right)+\frac{p_{t}^{\partial_{1} V}\left(X_{t}^{1}\right)}{p_{t}^{1}\left(X_{t}^{1}\right)} e_{1}\right) \mathrm{d} t+\sqrt{2} \mathrm{~d} W_{t},
$$

where $p_{t}$ is the unique solution to equation (3.2) in the sense of Definition 3.1. Notice that the drift

$$
b_{t}(x)=-\nabla V(x)+\frac{p_{t}^{\partial_{1} V}\left(x^{1}\right)}{p_{t}^{1}\left(x^{1}\right)} e_{1}
$$

in equation (3.17) is bounded, so that pathwise uniqueness holds (see [9]), as well as uniqueness in law, from the Girsanov Theorem.

\section{A Regularized approximate Dynamics}

To estimate the difference between the nonlinear process defined by equation (2.1) and its particle approximation (2.3), we introduce an intermediate process, called the regularized nonlinear process, which is the natural expected limit as $N$ goes to infinity of the particle approximation (2.3). The nonlinear term in this equation is more regular than the one in (2.1), so that we can show existence and uniqueness for this process. 
The aim of this section will be, in a first time, to prove existence and uniqueness for the regularized nonlinear process, see Theorem 4.1, and in a second time to show that the regularized nonlinear process converges to the nonlinear process solution to (2.1) as $\varepsilon$ and $\alpha$ go to zero, and to estimate the rate of this convergence, see Theorem 4.11 below. This will yield an existence result for the nonlinear process.

Under Assumption iv on the initial condition, for a fixed positive integer $n$, we expect the sequence of processes $\left(X_{n, N}^{\eta}\right)_{N>0}$ defined by $(2.3)$ to converge to a solution to

$$
\begin{cases}\mathrm{d} \bar{X}_{t, n}^{\eta} & =\left(-\nabla V\left(\bar{X}_{t, n}^{\eta}\right)+\frac{\varphi_{\eta} * P_{t}^{\eta, \partial_{1} V}}{\varphi_{\eta} * P_{t}^{\eta, 1}}\left(\bar{X}_{t, n}^{\eta, 1}\right) e_{1}\right) \mathrm{d} t+\sqrt{2} \mathrm{~d} W_{t}^{n}, \\ P_{t}^{\eta} & =\text { distribution of }\left\{\bar{X}_{t, n}^{\eta}\right\}\end{cases}
$$

with initial condition $\left(X_{0, n}\right)$.

\subsection{Existence and uniqueness for the regularized problem}

In this section, we show that pathwise uniqueness, uniqueness in distribution and strong existence hold for the regularized dynamics.

We first show existence and uniqueness of a solution to (4.1), using a fixed point method.

Theorem 4.1. Consider both the compact and the non compact cases. Under Assumptions i and iv, strong existence and uniqueness hold for equation (4.1).

Here we follow [15]: we show that a measure on the space of continuous paths from $[0, T]$ to $\mathbb{R}^{d}$ is the law of a solution to (4.1) if and only if it is a fixed point of some function $\Phi_{T}$. Then we show existence and uniqueness of this fixed point by a contraction argument. This cannot be done directly for equation (2.1), since its nonlinear term is too ill-behaved, so that we do not have contraction in that case.

For a probability measure $\mu$ on the set $\mathcal{C}_{T}=\mathcal{C}\left([0, T], \mathbb{R}^{d}\right)$ we denote by $\Phi_{T}(\mu)$ the distribution on $\mathcal{C}_{T}$ of the process $X$ defined by

$$
\mathrm{d} X_{t}=\left(-\nabla V\left(X_{t}\right)+\frac{\int_{\mathcal{C}_{T}} \varphi_{\eta}\left(X_{t}^{1}-x_{t}^{1}\right) \partial_{1} V\left(x_{t}\right) \mathrm{d} \mu(x)}{\int_{\mathcal{C}_{T}} \varphi_{\eta}\left(X_{t}^{1}-x_{t}^{1}\right) \mathrm{d} \mu(x)} e_{1}\right) \mathrm{d} t+\sqrt{2} \mathrm{~d} W_{t}
$$

whose initial condition $X_{0}$ has law $P_{0}$ and is independent of $W$. The distribution $\Phi_{T}(\mu)$ is well defined since equation (4.2), having global Lipschitz coefficients, has a unique strong solution.

Notice that, since

$$
\frac{\varphi_{\eta} * \mu_{t}^{\partial_{1} V}}{\varphi_{\eta} * \mu_{t}^{1}}=\frac{\int_{\mathcal{C}_{T}} \varphi_{\eta}\left(\cdot-x_{t}^{1}\right) \partial_{1} V\left(x_{t}\right) \mathrm{d} \mu(x)}{\int_{\mathcal{C}_{T}} \varphi_{\eta}\left(\cdot-x_{t}^{1}\right) \mathrm{d} \mu(x)},
$$

$\mu$ is the distribution of a solution to (4.1) up to time $T$ if, and only if $\Phi_{T}(\mu)=\mu$. We will show that such a $\mu$ exists and is unique using Picard's Theorem.

The Wasserstein metric $D_{T}\left(\mu_{1}, \mu_{2}\right)$ between two probability distributions $\mu_{1}$ and $\mu_{2}$ on $\mathcal{C}_{T}$ is defined by

$$
D_{T}\left(\mu_{1}, \mu_{2}\right)=\inf _{\pi \in \Pi} \int_{\mathcal{C}_{T} \times \mathcal{C}_{T}} 1 \wedge\|x-y\|_{\mathcal{C}_{T}} \mathrm{~d} \pi(x, y),
$$

where $\Pi=\left\{\pi \in \mathcal{P}\left(\mathcal{C}_{T} \times \mathcal{C}_{T}\right), \pi\right.$ having $\mu_{1}$ and $\mu_{2}$ as marginal distributions $\}$ is the set of all coupling of $\mu_{1}$ and $\mu_{2}$, and $\|\cdot\|_{\mathcal{C}_{T}}$ is the uniform norm on $\mathcal{C}_{T}$ :

$$
\|f-g\|_{\mathcal{C}_{T}}=\sup _{t \in[0, T]}|f(t)-g(t)| .
$$


More generally, for $t \in[0, T]$, we set

$$
D_{t}\left(\mu_{1}, \mu_{2}\right)=\inf _{\pi \in \Pi} \int_{\mathcal{C}_{T} \times \mathcal{C}_{T}} 1 \wedge \sup _{s \in[0, t]}\left|x_{s}-y_{s}\right| \mathrm{d} \pi(x, y) .
$$

Endowed with the Wasserstein metric, the space $\mathcal{P}\left(\mathcal{C}_{T}\right)$ of probability measures on $\mathcal{C}_{T}$ is complete. In order to apply a fixed point argument, we will need the following contraction lemma.

Lemma 4.2. Consider both the compact and non compact case. Let $T$ be a positive time. Under Assumption i, there is a positive constant $K$, not depending on $t$, satisfying

$$
D_{t}\left(\Phi_{T}\left(\mu_{1}\right), \Phi_{T}\left(\mu_{2}\right)\right) \leq K \int_{0}^{t} D_{s}\left(\mu_{1}, \mu_{2}\right) \mathrm{d} s,
$$

for all $t$ in $[0, T]$ and for all probability measures $\mu_{1}$ and $\mu_{2}$ in $\mathcal{P}\left(\mathcal{C}_{T}\right)$.

Proof. Let $\mu_{1}$ and $\mu_{2}$ be two probability measures on $\mathcal{C}_{T}$. For $i=1,2$, define $X_{t, i}$ by

$$
\mathrm{d} X_{t, i}=\left(-\nabla V\left(X_{t, i}\right)+\frac{\int_{\mathcal{C}_{T}} \varphi_{\eta}\left(X_{t, i}^{1}-x_{t}^{1}\right) \partial_{1} V\left(x_{t}\right) \mathrm{d} \mu_{i}(x)}{\int_{\mathcal{C}_{T}} \varphi_{\eta}\left(X_{t, i}^{1}-x_{t}^{1}\right) \mathrm{d} \mu_{i}(x)} e_{1}\right) \mathrm{d} t+\sqrt{2} \mathrm{~d} W_{t}
$$

with given initial condition $X_{0, i}=X_{0}$, for $i=1,2$.

Notice that

$$
\frac{\int_{\mathcal{C}_{T}} \varphi_{\eta}\left(\cdot-x_{t}^{1}\right) \partial_{1} V\left(x_{t}\right) \mathrm{d} \mu_{i}(x)}{\int_{\mathcal{C}_{T}} \varphi_{\eta}\left(\cdot-x_{t}^{1}\right) \mathrm{d} \mu_{i}(x)}=\frac{\varphi_{\eta} * \mu_{i, t}^{\partial_{1} V}}{\varphi_{\eta} * \mu_{i, t}^{1}},
$$

and that from (2.4) and Assumption i, the numerator and the denominator of (4.3) are respectively bounded from above and from below by positive constants depending only on $\eta$ and $V$. Then, for any $x, y$ and $0 \leq s \leq T$,

$$
\left|\frac{\varphi_{\eta} * \mu_{1, s}^{\partial_{1} V}}{\varphi_{\eta} * \mu_{1, s}^{1}}(x)-\frac{\varphi_{\eta} * \mu_{2, s}^{\partial_{1} V}}{\varphi_{\eta} * \mu_{2, s}^{1}}(y)\right| \leq K\left(|x-y| \wedge 1+D_{s}\left(\mu_{1}, \mu_{2}\right)\right) .
$$

Consequently,

$$
\mathbb{E}\left[1 \wedge\left\|X_{1}-X_{2}\right\|_{\mathcal{C}_{t}}\right] \leq K\left(\int_{0}^{t} \mathbb{E}\left[1 \wedge\left\|X_{1}-X_{2}\right\|_{\mathcal{C}_{s}}\right] \mathrm{d} s+\int_{0}^{t} D_{s}\left(\mu_{1}, \mu_{2}\right) \mathrm{d} s\right),
$$

for all $t \leq T$. Using Grönwall's Lemma, we then find, for any $t \leq T$,

But

$$
\mathbb{E}\left[1 \wedge\left\|X_{1}-X_{2}\right\|_{\mathcal{C}_{t}}\right] \leq K \int_{0}^{t} D_{s}\left(\mu_{1}, \mu_{2}\right) \mathrm{d} s .
$$

$$
D_{t}\left(\Phi_{t}\left(\mu_{1}\right), \Phi_{t}\left(\mu_{2}\right)\right) \leq \mathbb{E}\left[1 \wedge\left\|X_{1}-X_{2}\right\|_{\mathcal{C}_{t}}\right]
$$

since $X_{1}$ and $X_{2}$ respectively have $\Phi_{t}\left(\mu_{1}\right)$ and $\Phi_{t}\left(\mu_{2}\right)$ as distributions, finishing the proof.

Proof of Theorem 4.1. Iterating Lemma 4.2, we find existence and uniqueness of a fixed point of $\Phi_{T}$, given $X_{0}$, which yields uniqueness of the distribution $P$ of the solution to $(4.1)$ on $[0, T]$.

The law $P$ of any solution being unique, we can substitute the marginal of $P$ at time $t$ in equation (4.1), and we obtain a linear stochastic differential equation with Lipschitz continuous coefficients. Pathwise uniqueness holds for that kind of equation, so that weak existence and pathwise uniqueness hold for (4.1). Consequently, from Yamada-Watanabe Theorem, it admits a unique strong solution. 


\subsection{Convergence to the nonlinear process}

We are now going to let $\varepsilon$ and $\alpha$ go to 0 in (4.1).

We denote by $X_{t}^{\eta}$ the unique strong solution to (4.1), with initial condition $X_{0}$ and Brownian motion $W^{n}$ replaced with $W$. The distribution of $\left\{X_{t}^{\eta}\right\}$ will be denoted $P^{\eta}$. We expect a possible limit $X$ of $X^{\eta}$ as $\eta$ goes to 0 to be a solution to (2.1). To this aim, we define the following martingale problem:

Definition 4.3. We say that a probability measure $P$ on the space $\mathcal{C}_{T}$ of continuous paths is a solution to the martingale problem associated to (2.1) if its time marginals $P_{t}$ admit a density $p_{t}$ with respect to the Lebesgue measure, and if, under the measure $P$,

- the canonical process $x \in \mathcal{C}_{T}$ is such that for any twice differentiable function which is bounded as well as its first and second derivatives, the process

$$
m_{t}=\psi\left(x_{t}\right)-\psi\left(x_{0}\right)+\int_{0}^{t} \nabla \psi\left(x_{s}\right) \nabla V\left(x_{s}\right) \mathrm{d} s-\int_{0}^{t} \Delta \psi\left(x_{s}\right) \mathrm{d} s-\int_{0}^{t} \partial_{1} \psi\left(x_{s}\right) \frac{p_{t}^{\partial_{1} V}\left(x_{t}\right)}{p_{t}^{1}\left(x_{t}\right)} \mathrm{d} s,
$$

is a martingale with respect to the filtration $\sigma\left(x_{s}, s \leq t\right)$.

- $\left\{x_{0}\right\}$ has law $P_{0}$.

Notice that, since the drift coefficient is bounded, the Girsanov Theorem shows that it is not restrictive to assume that $P_{t}$ has a density.

We deduce from Theorem 3.8 the following result:

Proposition 4.4. In the compact case under Assumptions $\mathrm{i}$ and ii, or in the non compact case under Assumptions i-iii, uniqueness holds for the martingale problem defined in Definition 4.3.

Our aim in this section will be to prove the following results:

Theorem 4.5. Let Assumptions i and ii hold.

In the compact case, $\left(P^{\eta}\right)_{\eta>0}$ converges as $\eta$ goes to 0 to the solution of the martingale problem.

In the non compact case, the family of probability measures $\left(P^{\eta}\right)_{\eta>0}$ is tight, and any converging subsequence converges to a solution of the martingale problem defined in Definition 4.3. Under the additional Assumption iii, $\left(P_{\eta}\right)_{\eta>0}$ actually converges to the unique solution.

As a corollary of Theorem 4.5, one has existence of solutions to (2.1) (under regularity assumptions on the initial condition).

From Proposition 4.4, in order to prove Theorem 4.5, it is enough to prove that the family $\left(P^{\eta}\right)_{\eta>0}$ is tight, and that any converging subsequence converges to a solution of the martingale problem.

Our first step will be to derive the Fokker-Planck equation satisfied by the distribution of $\left\{X_{t}^{\eta}\right\}$. Let $\psi$ be a smooth bounded function on $\mathcal{D}$, with bounded derivatives. Applying Itō's formula to $\psi\left(X_{t}^{\eta}\right)$ and taking the expectation, we find that

$$
\int_{\mathcal{D}} \psi \mathrm{d} P_{T}^{\eta}=\int_{\mathcal{D}} \psi p_{0}(x) \mathrm{d} x+\int_{0}^{T} \int_{\mathcal{D}}(\Delta \psi-\nabla \psi \cdot \nabla V) \mathrm{d} P_{t}^{\eta} \mathrm{d} t+\int_{0}^{T} \int_{\mathcal{D}} \partial_{1} \psi \frac{\varphi_{\eta} * P_{t}^{\eta, \partial_{1} V}}{\varphi_{\eta} * P_{t}^{\eta, 1}} \mathrm{~d} P_{t}^{\eta} \mathrm{d} t
$$

Equation (4.5) is a weak formulation of the following partial differential equation

$$
\partial_{t} P_{t}^{\eta}=\operatorname{div}\left(P_{t}^{\eta} \nabla V+\nabla P_{t}^{\eta}\right)-\partial_{1}\left(P_{t}^{\eta} \frac{\varphi_{\eta} * P_{t}^{\eta, \partial_{1} V}}{\varphi_{\eta} * P_{t}^{\eta, 1}}\right)
$$

We are going to show that $P_{t}^{\eta}$, or more precisely, its density, is actually a solution to equation (4.6) in the following stronger sense. 
Definition 4.6. A function $u$ is said to be a solution to (4.6) with initial condition $p_{0}$ if, in the compact case,

- $u$ belongs to $\mathbb{L}^{\infty}\left((0, T), \mathbb{L}^{2}\left(\mathbb{T}^{d}\right)\right) \bigcap \mathbb{L}^{2}\left((0, T), \mathbb{H}^{1}\left(\mathbb{T}^{d}\right)\right)$;

- for any function $\psi \in \mathbb{H}^{1}\left(\mathbb{T}^{d}\right)$, we have:

$$
\partial_{t} \int_{\mathcal{D}} u_{t} \psi=-\int_{\mathcal{D}} u_{t} \nabla V \cdot \nabla \psi-\int_{\mathcal{D}} \nabla u_{t} \cdot \nabla \psi+\int_{\mathcal{D}}\left(\partial_{1} \psi\right) u_{t} \frac{\varphi_{\eta} * u_{t}^{\partial_{1} V}}{\varphi_{\eta} * u_{t}^{1}}
$$

in the sense of distributions in time;

- $u_{0}=p_{0}$.

In the non compact case these conditions are replaced by

- $u$ belongs to $\mathbb{L}^{\infty}\left((0, T), \mathbb{L}^{2}(w)\right) \cap \mathbb{L}^{2}\left((0, T), \mathbb{H}^{1}(w)\right)$;

- for any function $\psi \in \mathbb{H}^{1}(w)$, we have:

$\partial_{t} \int_{\mathcal{D}} u_{t} \psi w=-\int_{\mathcal{D}} u_{t} \nabla V \cdot(w \nabla \psi+\psi \nabla w)-\int_{\mathcal{D}} \nabla u_{t} \cdot(w \nabla \psi+\psi \nabla w)+\int_{\mathcal{D}}\left(\partial_{1} \psi\right) u_{t} \frac{\varphi_{\eta} * u_{t}^{\partial_{1} V}}{\varphi_{\eta} * u_{t}^{1}} w$

in the sense of distributions in time;

- $u_{0}=p_{0}$.

As for Definition 3.1, these conditions make sense.

With this definition, one has the following result:

Lemma 4.7. Consider both the compact and the non compact cases. Under Assumptions i and ii, the distribution $P_{t}^{\eta}$ of $\left\{X_{t}^{\eta}\right\}$ has a density $p_{t}^{\eta}$ with respect to the Lebesgue measure such that $p^{\eta}$ satisfies (4.6) in the sense of Definition 4.6.

Moreover, the family $\left(p^{\eta}\right)_{\eta>0}$ is bounded in $\mathbb{L}^{\infty}\left((0, T), \mathbb{L}^{2}(\mathcal{D})\right) \cap \mathbb{L}^{2}\left((0, T), \mathbb{H}^{1}(\mathcal{D})\right)$ and, in the non compact case, under Assumption iii, $\left(p^{\eta}\right)_{\eta>0}$ is bounded in $\mathbb{L}^{\infty}\left((0, T), \mathbb{L}^{2}(w)\right) \cap \mathbb{L}^{2}\left((0, T), \mathbb{H}^{1}(w)\right)$.

Proof. Since the drift coefficient in (4.1) is bounded, following the proof of Lemmas 3.3 and 3.4, we obtain that $P_{t}^{\eta}$ has a density $p_{t}^{\eta}$, where $p^{\eta}$ satisfies the first condition in Definition 4.6. Applying Itō's formula to $\psi\left(X_{t}^{\eta}\right)$ for some smooth $\psi$, we find that (4.7) ((4.8) in the non compact case) holds for a smooth $\psi$. Using the density of smooth functions in $\mathbb{H}^{1}\left(\mathbb{T} \times \mathbb{R}^{d-1}\right)$, it holds for any $\psi$ in $\mathbb{H}^{1}\left(\mathbb{T}^{d}\right)$, and the same is true for $\mathbb{H}^{1}(w)$ in the non compact case.

To prove that $p^{\eta}$ is bounded independently of $\eta$, notice that from the boundedness of $\nabla V$, the function $\frac{\varphi_{\eta} * *_{t}^{\eta, \partial_{1} V}}{\varphi_{\eta} * p_{t}^{\eta, 1}}$ is bounded from above uniformly with respect to $\eta$. Consequently, from Cauchy-Schwarz inequality,

$$
\begin{aligned}
\frac{1}{2} \partial_{t}\left\|p_{t}^{\eta}\right\|_{\mathbb{L}^{2}\left(\mathbb{T} \times \mathbb{R}^{d-1}\right)}^{2} & =-\left\|\nabla p_{t}^{\eta}\right\|_{\mathbb{L}^{2}\left(\mathbb{T} \times \mathbb{R}^{d-1}\right)}^{2}-\int_{\mathbb{T} \times \mathbb{R}^{d-1}} p_{t}^{\eta} \nabla p_{t}^{\eta} \cdot \nabla V+\int_{\mathbb{T} \times \mathbb{R}^{d-1}}\left(\partial_{1} p_{t}^{\eta}\right) p_{t}^{\eta} \frac{\varphi_{\eta} * p_{t}^{\eta, \partial_{1} V}}{\varphi_{\eta} * p_{t}^{\eta, 1}} \\
& \leq-\left\|\nabla p_{t}^{\eta}\right\|_{\mathbb{L}^{2}\left(\mathbb{T} \times \mathbb{R}^{d-1}\right)}^{2}+K\left\|p_{t}^{\eta}\right\|_{\mathbb{L}^{2}\left(\mathbb{T} \times \mathbb{R}^{d-1}\right)}\left\|\nabla p_{t}^{\eta}\right\|_{\mathbb{L}^{2}\left(\mathbb{T} \times \mathbb{R}^{d-1}\right)}
\end{aligned}
$$

where the constant $K$ does not depend on $\eta$. We finish the proof using Young's inequality, and then Grönwall's Lemma.

The proof is similar in the non compact case.

Thanks to Lemma 4.7, we can prove the relative compactness of the family $p^{\eta}$ in a nice sense.

Lemma 4.8. Consider both the compact and the non compact cases. Under Assumptions i and ii, for any bounded open domain $\mathcal{O}$ in $\mathcal{D}$, the set $\left(p^{\eta} \mid \mathcal{O}\right)_{\eta>0}$ of restrictions of the functions $p^{\eta}$ to $\mathcal{O}$ is relatively compact in the space $\mathbb{L}^{2}\left((0, T), \mathbb{L}^{2}(\mathcal{O})\right)$. Moreover, the set $\left(P^{\eta}\right)_{\eta>0}$ of laws of the solution is tight. 
Proof. We first prove the relative compactness of $p^{\eta}$ in $\mathbb{L}^{2}\left((0, T), \mathbb{L}^{2}(\mathcal{O})\right)$. We use the fact that for a bounded open domain $\mathcal{O}$ and for $p, q \in(1, \infty)$, the space

$$
E_{p, q}=\left\{f \in \mathbb{L}^{p}\left((0, T), \mathbb{H}^{1}(\mathcal{O})\right) \text {, such that } \partial_{t} f \in \mathbb{L}^{q}\left((0, T), \mathbb{H}^{-1}(\mathcal{O})\right)\right\}
$$

imbeds compactly in $\mathbb{L}^{p}\left((0, T), \mathbb{L}^{2}(\mathcal{O})\right)$ (see [12], p. 57). We already know that the set $\left(p^{\eta}\right)_{\eta>0}$ is bounded in $\mathbb{L}^{2}\left((0, T), \mathbb{H}^{1}(\mathcal{D})\right)$, so that the set $\left(p^{\eta} \mid \mathcal{O}\right)_{\eta>0}$ is bounded in $\mathbb{L}^{2}\left((0, T), \mathbb{H}^{1}(\mathcal{O})\right)$. Thus, it is enough to show that $\left(\partial_{t} p^{\eta} \mid \mathcal{O}\right)_{\eta>0}$ is bounded in $\mathbb{L}^{q}\left((0, T), \mathbb{H}^{-1}(\mathcal{O})\right)$, for some $q \in(1, \infty)$ to finish the proof. The following equation holds

$$
\partial_{t} p^{\eta}=\operatorname{div}\left(p^{\eta} \nabla V\right)+\Delta p^{\eta}-\partial_{1}\left(p^{\eta} \frac{\varphi_{\eta} * p_{t}^{\eta, \partial_{1} V}}{\varphi_{\eta} * p_{t}^{\eta, 1}}\right),
$$

showing, since $\left(p^{\eta}\right)_{\eta>0}$ is bounded in $\mathbb{L}^{2}\left((0, T), \mathbb{H}^{1}(\mathcal{D})\right)$, that $\left(\partial_{t} p^{\eta}\right)_{\eta>0}$ is bounded in $\mathbb{L}^{2}\left((0, T), \mathbb{H}^{-1}(\mathcal{D})\right)$, thus, $\partial_{t} p^{\eta}{ }_{\mathcal{O}}$ is bounded in $\mathbb{L}^{2}\left((0, T), \mathbb{H}^{-1}(\mathcal{O})\right)$. This shows that $\left(p_{\mid \mathcal{O}}^{\eta}\right)_{\eta>0}$ is relatively compact in $\mathbb{L}^{2}\left((0, T), \mathbb{L}^{2}(\mathcal{O})\right)$.

Now we prove the relative compactness of $\left(P^{\eta}\right)_{\eta>0}$ in $\mathcal{P}\left(\mathcal{C}_{T}\right)$. For this aim, we use Kolmogorov compactness criterion. At time $t=0, X_{0}^{\eta}$ is equal to $X_{0}$, independently of $\eta$. Consequently, the family $\left(X_{0}^{\eta}\right)_{\eta>0}$ is tight. To conclude the proof, it is enough to show that for some positive constants $a, b$ and $K$,

$$
\sup _{\eta>0} \mathbb{E}\left[\left|X_{t}^{\eta}-X_{s}^{\eta}\right|^{a}\right] \leq K|t-s|^{1+b}
$$

for $0 \leq s, t \leq T$. Since $\nabla V$ is bounded, we have, for $0 \leq s, t \leq T$ and $p>1$,

$$
\begin{aligned}
\mathbb{E}\left[\left|X_{t}^{\eta}-X_{s}^{\eta}\right|^{p}\right]^{1 / p} & \leq \mathbb{E}\left[\left|\int_{s}^{t} \nabla V\left(X_{\tau}^{\eta}\right) \mathrm{d} \tau\right|^{p}\right]^{1 / p}+\mathbb{E}\left[\left|W_{t}-W_{s}\right|^{p}\right]^{1 / p}+\mathbb{E}\left[\left|\int_{s}^{t} \frac{\varphi_{\eta} * u_{\tau}^{\eta, \partial_{1} V}\left(X_{\tau}^{\eta, 1}\right)}{\varphi_{\eta} * u_{\tau}^{\eta, 1}\left(X_{\tau}^{\eta, 1}\right)} \mathrm{d} \tau\right|^{p}\right]^{1 / p} \\
& \leq K\left(|t-s|+|t-s|^{1 / 2}\right) .
\end{aligned}
$$

This rewrites

$$
\mathbb{E}\left[\left|X_{t}^{\eta}-X_{s}^{\eta}\right|^{p}\right] \leq K|t-s|^{p / 2},
$$

for some positive $K$. Taking $p=3$, Lemma 4.8 follows.

As a consequence of Lemma 4.8, using a diagonal argument, we can extract a subsequence of $\eta \rightarrow 0$, still denoted $\eta$ such that:

- $p^{\eta}$ converges almost everywhere on $(0, T) \times \mathcal{D}$ and in $\mathbb{L}^{2}\left((0, T), \mathbb{L}^{2}(\mathcal{O})\right)=\mathbb{L}^{2}((0, T) \times \mathcal{O})$ as $\eta$ goes to 0 , for any bounded open domain $\mathcal{O}$ to a function $p^{0}$.

- $P^{\eta}$ converges in $\mathcal{P}(\mathcal{C}([0, T]))$ as $\eta$ goes to 0 to a probability measure $P^{0}$.

To let $\eta$ go to zero in (4.1), we finally need the following lemma.

Lemma 4.9. Consider both the compact and the non compact cases. Under Assumptions $\mathrm{i}$ and ii, the limit $p^{0}$ of $p^{\eta}$ is such that $p_{t}^{0}$ is the density of the time marginal of $P^{0}$ for almost all times $t$. Moreover, the convergence of $p^{\eta}$ to $p^{0}$ also holds in $\mathbb{L}^{1}((0, T) \times \mathcal{D})$ and up to a second subsequence extraction, $\frac{\varphi_{\eta} * p^{\eta, \partial_{1} V}}{\varphi_{\eta} * p^{\eta, 1}}$ converges almost everywhere on $(0, T) \times \mathbb{T}$ to $\frac{p^{0, \partial_{1} V}}{p^{0,1}}$ as $\eta$ goes to zero.

Proof. We first prove that $p^{\eta}$ converges to $p^{0}$ in $\mathbb{L}^{1}((0, T) \times \mathcal{D})$. It holds

$$
\int_{0}^{T} \int_{\mathcal{D}}\left|p^{\eta}-p^{0}\right|=\int_{0}^{T} \int_{\mathcal{D}}\left(p^{\eta}-p^{0}\right)+2 \int_{0}^{T} \int_{\mathcal{D}}\left(p^{\eta}-p^{0}\right)^{-}=2 \int_{0}^{T} \int_{\mathcal{D}}\left(p^{\eta}-p^{0}\right)^{-} .
$$


But $p^{\eta}$ converges almost everywhere to $p^{0}$, and $\left(p^{\eta}-p^{0}\right)^{-}$is bounded from above by the integrable function $p^{0}$. Consequently, by the Lebesgue Theorem, $p^{\eta}$ converges to $p^{0}$ in $\mathbb{L}^{1}((0, T) \times \mathcal{D})$.

A consequence of this convergence and of the boundedness of $\partial_{1} V$ is that the sequences $\left(p^{\eta, \partial_{1} V}\right)_{\eta>0}$ and $\left(p^{\eta, 1}\right)_{\eta>0}$ converge in $\mathbb{L}^{1}((0, T) \times \mathbb{T})$ respectively to $p^{0, \partial_{1} V}$ and $p^{0,1}$.

As a consequence, $\varphi_{\eta} * p^{\eta, 1}$ and $\varphi_{\eta} * p^{\eta, \partial_{1} V}$ also converge in $\mathbb{L}^{1}((0, T) \times \mathbb{T})$ to the same limits. Therefore, up to the extraction of a second subsequence, we have pointwise convergence almost everywhere for the denominator and the numerator of $\frac{\varphi_{\eta} * p^{\eta, \partial_{1} V}}{\varphi_{\eta} * p^{\eta, 1}}$.

Now we show that $p_{t}^{0}$ is for almost all $t$ the density of the time marginal $P_{t}^{0}$ of $P^{0}$. Since $P^{\eta}$ converges to $P^{0}$ as $\eta$ goes to 0 in $\mathcal{P}\left(\mathcal{C}_{T}\right)$, then $\mathbb{E}\left[\Psi\left(X^{\eta}\right)\right]$ converges to $\mathbb{E}\left[\Psi\left(X^{0}\right)\right]$ as $\eta$ goes to 0 , for any bounded continuous functional $\Psi$ on $\mathcal{C}_{T}$. Taking a function of the form $\Psi(Y)=\int_{0}^{T} \theta(t) \tilde{\Psi}\left(Y_{t}\right) \mathrm{d} t$ where $\tilde{\Psi}$ and $\theta$ are bounded and continuous, one has

$$
\mathbb{E}\left[\Psi\left(X^{\eta}\right)\right]=\int_{0}^{T} \theta(t)\left(\int_{\mathbb{T}^{\prime} \times \mathbb{R}^{d-1}} \tilde{\Psi} p_{t}^{\eta}\right) \mathrm{d} t .
$$

Moreover, since $p^{\eta}$ converges to $p^{0}$ in $\mathbb{L}^{1}\left((0, T) \times \mathbb{T} \times \mathbb{R}^{d-1}\right)$, one has

$$
\int_{0}^{T}\left(\theta(t) \int_{\mathbb{T} \times \mathbb{R}^{d-1}} \tilde{\Psi} p_{t}^{\eta}\right) \mathrm{d} t \rightarrow_{\eta \rightarrow 0} \int_{0}^{T} \theta(t)\left(\int_{\mathbb{T} \times \mathbb{R}^{d-1}} \tilde{\Psi} p_{t}^{0}\right) \mathrm{d} t .
$$

As a result,

$$
\mathbb{E}\left[\int_{0}^{T} \theta(t) \tilde{\Psi}\left(X_{t}^{0}\right) \mathrm{d} t\right]=\int_{0}^{T} \theta(t)\left(\int_{\mathbb{T} \times \mathbb{R}^{d-1}} \tilde{\Psi} p_{t}^{0}\right) \mathrm{d} t,
$$

so that, almost everywhere, $p_{t}^{0}$ is the time marginal of $P^{0}$.

We can now prove Theorem 4.5. We want to prove that $P^{0}$ is a solution to the martingale problem defined in Definition 4.3. It is enough to show that for $0 \leq s_{1} \leq \ldots \leq s_{n} \leq s \leq t$, any bounded continuous function $g$ and any twice differentiable function $\psi$ with bounded derivatives, one has $\int_{\mathcal{C}_{T}} g\left(x_{s_{1}}, \ldots, x_{s_{n}}\right)\left(m_{t}-m_{s}\right) \mathrm{d} P^{0}=0$.

Under the probability measure $P^{\eta}$, the canonical process $x \in \mathcal{C}([0, T])$ is such that

$$
m_{t}^{\eta}=\psi\left(x_{t}\right)-\psi\left(x_{0}\right)-\int_{0}^{t} \Delta \psi\left(x_{s}\right) \mathrm{d} s+\int_{0}^{t} \nabla V\left(x_{s}\right) \nabla \psi\left(x_{s}\right) \mathrm{d} s-\int_{0}^{t} \partial_{1} \psi\left(x_{s}\right) \frac{\varphi_{\eta} * p_{s}^{\eta, \partial_{1} V}\left(x_{s}^{1}\right)}{\varphi_{\eta} * p_{s}^{\eta, 1}\left(x_{s}^{1}\right)} \mathrm{d} s
$$

is a martingale. We thus have

$$
\int_{\mathcal{C}_{T}} g\left(x_{s_{1}}, \ldots, x_{s_{n}}\right)\left(m_{t}^{\eta}-m_{s}^{\eta}\right) \mathrm{d} P^{\eta}=0 .
$$

Consequently, denoting $\tilde{\eta}=(\tilde{\varepsilon}, \tilde{\alpha})$

$$
\begin{aligned}
\left|\int_{\mathcal{C}_{T}} g\left(x_{s_{1}}, \ldots, x_{s_{n}}\right)\left(m_{t}-m_{s}\right) \mathrm{d} P^{0}\right| \leq & \left|\int_{\mathcal{C}_{T}} g\left(x_{s_{1}}, \ldots, x_{s_{n}}\right)\left(m_{t}^{\tilde{\eta}}-m_{s}^{\tilde{\eta}}\right) \mathrm{d} P^{\eta}\right| \\
& +\left|\int_{\mathcal{C}_{T}} g\left(x_{s_{1}}, \ldots, x_{s_{n}}\right)\left(m_{t}^{\tilde{\eta}}-m_{s}^{\tilde{\eta}}\right) \mathrm{d}\left(P^{\eta}-P^{0}\right)\right| \\
& +\left|\int_{\mathcal{C}_{T}} g\left(x_{s_{1}}, \ldots, x_{s_{n}}\right)\left(\left(m_{t}^{\tilde{\eta}}-m_{s}^{\tilde{\eta}}\right)-\left(m_{t}-m_{s}\right)\right) \mathrm{d} P^{0}\right| .
\end{aligned}
$$

Taking $\lim \sup _{\tilde{\eta} \rightarrow 0} \lim \sup _{\eta \rightarrow 0}$, we obtain:

$$
\left|\int_{\mathcal{C}_{T}} g\left(x_{s_{1}}, \ldots, x_{s_{n}}\right)\left(m_{t}-m_{s}\right) \mathrm{d} P^{0}\right| \leq \lim \sup _{\tilde{\eta} \rightarrow 0} \lim \sup _{\eta \rightarrow 0}\left|\int_{\mathcal{C}_{T}} g\left(x_{s_{1}}, \ldots, x_{s_{n}}\right)\left(m_{t}^{\tilde{\eta}}-m_{s}^{\tilde{\eta}}\right) \mathrm{d} P^{\eta}\right| .
$$


Indeed, $g\left(x_{s_{1}}, \ldots, x_{s_{n}}\right)\left(m_{t}^{\tilde{\eta}}-m_{s}^{\tilde{\eta}}\right)$ is a bounded continuous function of $x$, and $P^{\eta}$ converges to $P^{0}$. Moreover, we have

$$
\begin{aligned}
& \left|\int_{\mathcal{C}_{T}} g\left(x_{s_{1}}, \ldots, x_{s_{n}}\right)\left(\left(m_{t}^{\tilde{\eta}}-m_{s}^{\tilde{\eta}}\right)-\left(m_{t}-m_{s}\right)\right) \mathrm{d} P^{0}\right| \\
& =\left|\int_{\mathcal{C}_{T}} \int_{s}^{t} g\left(x_{s_{1}}, \ldots, x_{s_{2}}\right) \partial_{1} \psi\left(x_{\tau}\right)\left[\frac{\varphi_{\tilde{\eta}} * p_{\tau}^{\tilde{\eta}, \partial_{1} V}}{\varphi_{\tilde{\eta}} * p_{\tau}^{\tilde{\eta}, 1}}-\frac{p_{\tau}^{0, \partial_{1} V}}{p_{\tau}^{0,1}}\right]\left(x_{\tau}^{1}\right) \mathrm{d} \tau \mathrm{d} P^{0}(x)\right| \\
& \leq K \int_{s}^{t} \int_{\mathcal{D}}\left|\left[\frac{\varphi_{\tilde{\eta}} * p_{\tau}^{\tilde{\eta}, \partial_{1} V}}{\varphi_{\tilde{\eta}} * p_{\tau}^{\tilde{\eta}, 1}}-\frac{p_{\tau}^{0, \partial_{1} V}}{p_{\tau}^{0,1}}\right](y)\right| p_{\tau}^{0}(y) \mathrm{d} y \mathrm{~d} \tau .
\end{aligned}
$$

This last integral goes to 0 as $\tilde{\eta}$ goes to 0 , since the function $\left[\frac{\varphi_{\eta} * p_{\tau}^{\tilde{\eta}, \partial_{1} V}}{\varphi_{\tilde{\eta}} * p_{\tau}^{\tilde{\eta}, 1}}-\frac{p_{\tau}^{0, \partial_{1} V}}{p_{\tau}^{0,1}}\right]$ converges almost everywhere to 0 on $[s, t] \times \mathcal{D}$, and is bounded from above by some positive constant. To conclude, we estimate the right hand side in (4.9):

$$
\begin{aligned}
& \left|\int_{\mathcal{C}_{T}} g\left(x_{s_{1}}, \ldots, x_{s_{n}}\right)\left(m_{t}^{\tilde{\eta}}-m_{s}^{\tilde{\eta}}\right) \mathrm{d} P^{\eta}(x)\right| \\
& =\left|\int_{\mathcal{C}_{T}} g\left(x_{s_{1}}, \ldots, x_{s_{n}}\right)\left(\left(m_{t}^{\tilde{\eta}}-m_{s}^{\tilde{\eta}}\right)-\left(m_{t}^{\eta}-m_{s}^{\eta}\right)\right) \mathrm{d} P^{\eta}(x)\right| \\
& =\left|\int_{\mathcal{C}_{T}} g\left(x_{s_{1}}, \ldots, x_{s_{n}}\right) \int_{s}^{t} \partial_{1} \psi\left(x_{\tau}\right)\left(\frac{\varphi_{\eta} * p_{\tau}^{\eta, \partial_{1} V}}{\varphi_{\eta} * p_{\tau}^{\eta, 1}}-\frac{\varphi_{\tilde{\eta}} * p_{\tau}^{\tilde{\eta}, \partial_{1} V}}{\varphi_{\tilde{\eta}} * p_{\tau}^{\tilde{\eta}, 1}}\right)\left(x_{\tau}^{1}\right) \mathrm{d} \tau \mathrm{d} P^{\eta}(x)\right| \\
& \leq K \int_{s}^{t} \int_{\mathcal{C}_{T}}\left|\frac{\varphi_{\eta} * p_{\tau}^{\eta, \partial_{1} V}\left(x_{\tau}^{1}\right)}{\varphi_{\eta} * p_{\tau}^{\eta, 1}\left(x_{\tau}^{1}\right)}-\frac{\varphi_{\tilde{\eta}} * p_{\tau}^{\tilde{\eta}, \partial_{1} V}\left(x_{\tau}^{1}\right)}{\varphi_{\tilde{\eta}} * p_{\tau}^{\tilde{\eta}, 1}\left(x_{\tau}^{1}\right)}\right| \mathrm{d} P^{\eta}(x) \mathrm{d} \tau \\
& =K \int_{s}^{t} \int_{\mathbb{T}}\left|\frac{\varphi_{\eta} * p_{\tau}^{\eta, \partial_{1} V}(y)}{\varphi_{\eta} * p_{\tau}^{\eta, 1}(y)}-\frac{\varphi_{\tilde{\eta}} * p_{\tau}^{\tilde{\eta}, \partial_{1} V}(y)}{\varphi_{\tilde{\eta}} * p_{\tau}^{\tilde{\eta}, 1}(y)}\right| p_{\tau}^{\eta, 1}(y) \mathrm{d} y \mathrm{~d} \tau .
\end{aligned}
$$

This last integral tends to 0 as $\eta$ and $\tilde{\eta}$ go to 0 , since $p^{\eta, 1}$ converges in $\mathbb{L}^{1}((s, t) \times \mathbb{T})$, and since $\frac{\varphi_{\eta} * p_{\tau}^{\eta, \partial_{1} V}(y)}{\varphi_{\eta} * p_{\tau}^{\eta, 1}(y)}-$ $\frac{\varphi_{\tilde{\eta}} * p_{\tau}^{\tilde{\eta}, \partial_{1} V}(y)}{\varphi_{\tilde{\eta}} * p_{\tau}^{\tilde{\eta}, 1}(y)}$ is bounded and converges almost everywhere to 0 . We then obtain Theorem 4.5.

\subsection{Another existence result for the nonlinear process}

From Theorem 4.5, we know that existence holds for (2.1) under some regularity assumptions on the initial condition. Indeed, if $P^{0}$ is the limit of some subsequence of $P^{\eta}$, then the canonical process $x$ defined on the canonical space $\left(\mathcal{C}_{T}, P^{0}\right)$ is a solution to equation (2.1). By approximating the initial condition by regular densities, one can relax the regularity assumption.

Theorem 4.10. Consider both the compact and non compact cases. Under Assumption i, weak existence holds for equation (2.1) with given initial condition $X_{0}$. Moreover, for positive s, the law of $\left\{X_{s}\right\}$ has a density $p_{s}$ such that, for $0<t<T$,

$$
p \in \mathbb{L}^{\infty}\left((t, T), \mathbb{L}^{2}(\mathcal{D})\right) \bigcap \mathbb{L}^{2}\left((t, T), \mathbb{H}^{1}(\mathcal{D})\right) .
$$

Notice that, under the hypotheses of Theorem 4.10, we have no uniqueness result.

Proof. Theorem 4.5 yields existence for (2.1) when the initial condition satisfies Assumption ii. To prove existence without assumption on the initial condition, we use approximations of the initial condition. Let $\left(p_{0}^{k}\right)_{k \in \mathbb{N}}$ be a sequence of probability densities satisfying Assumption ii and converging to $p^{0}$ in $\mathcal{P}(\mathcal{D})($ for example, 
this sequence can be obtained by convolution with a Gaussian kernel). From Theorem 4.5, there exists a solution $\left(X_{t}^{k}\right)$ to equation $(2.1)$ driven by a Brownian motion $W$ defined on some probability space $(\Omega, \mathcal{F}, \mathbb{P})$, such that $X_{0}^{k}$ admits $p_{0}^{k}$ as density.

As in the proof of Lemma 4.8, we can apply Kolmogorov criterion, so that the family of distributions $P^{k}$ of $\left(\left\{X_{t}^{k}\right\}\right)_{0 \leq t \leq T}$ is tight. Consequently, we can extract from $\left(P^{k}\right)$ a converging subsequence whose limit is denoted $P$. To prove that $P$ satisfies the martingale problem defined in Definition 4.3, we need some estimate on the time marginals of $P^{k}$, uniformly in $k$.

According to Lemma 3.4, the law of $\left\{X_{t}^{k}\right\}$ has a density $p_{t}^{k}$ such that $p^{k}$ lies in $\mathbb{L}^{\infty}\left((0, T), \mathbb{L}^{2}(\mathcal{D})\right)$ and $\mathbb{L}^{2}\left((0, T), \mathbb{H}^{1}(\mathcal{D})\right)$. Notice that the drift coefficient $b_{t}^{k}\left(X_{t}\right) \stackrel{\text { def }}{=}-\nabla V\left(X_{t}\right)+\mathbb{E}\left[\partial_{1} V\left(X_{t}\right) \mid\left\{X_{t}^{1}\right\}\right] e_{1}$ in equation $(2.1)$ is bounded, so that we can apply the Girsanov Theorem. Indeed, define

$$
L_{t}^{k}=\exp \left(-\frac{1}{\sqrt{2}} \int_{0}^{t} b_{s}^{k}\left(X_{s}^{k}\right) \mathrm{d} W_{s}-\frac{1}{4} \int_{0}^{t}\left\|b_{s}^{k}\left(X_{s}^{k}\right)\right\|^{2} \mathrm{~d} s\right)
$$

Novikov's Condition is satisfied for this process, so that the formula

$$
\mathbb{Q}_{k}(A)=\mathbb{E}\left[\mathbf{1}_{A} L_{t}^{k}\right]
$$

for $A \in \sigma\left(W_{s}\right)_{s \leq t}$, defines a probability distribution $\mathbb{Q}_{k}$ on $\Omega$ such that, under $\mathbb{Q}_{k}$, the process

$$
\frac{1}{\sqrt{2}}\left(X_{t}^{k}-X_{0}^{k}\right)=W_{t}+\frac{1}{\sqrt{2}} \int_{0}^{t} b_{s}^{k}\left(X_{s}^{k}\right) \mathrm{d} s
$$

is a Brownian motion. Denote $\gamma_{t}^{k}$ the law of $\left\{X_{t}^{k}\right\}$ under $\mathbb{Q}_{k}$. Notice that since, under $\mathbb{Q}_{k}, X_{t}^{k}$ is the sum of $\sqrt{2}$ times a Brownian motion at time $t$ and an independent random variable $X_{0}^{k}, \gamma_{t}^{k}$ has a density with respect to the Lebesgue measure which is bounded by $\frac{K}{t^{d / 2}}$ where $K$ is a constant not depending on $k$ and $t$. As a result, for a given function $\psi$ in $\mathbb{L}^{2}(\mathcal{D})$, one has

$$
\left|\int_{\mathcal{D}} \psi(x) \mathrm{d} P_{t}^{k}(x)\right|=\left|\mathbb{E}\left[\psi\left(X_{t}^{k}\right)\right]\right|=\left|\mathbb{E}_{\mathbb{Q}_{k}}\left[\psi\left(X_{t}^{k}\right)\left(L_{t}^{k}\right)^{-1}\right]\right| \leq\left(\int_{\mathcal{D}} \psi^{2} \mathrm{~d} \gamma_{t}^{k}\right)^{\frac{1}{2}} \mathbb{E}\left[\left(L_{t}^{k}\right)^{-2}\right]^{1 / 2} \leq \frac{K}{t^{d / 4}}\|\psi\|_{\mathbb{L}^{2}(\mathcal{D})},
$$

where $K$ is a positive constant, which does not depend on $k$ since $\left|b^{k}\right|$ is bounded from above by $\|\nabla V\|_{\mathbb{L}^{\infty}}$. Consequently, for any $0<t<T,\left\|p_{s}^{k}\right\|_{\mathbb{L}^{2}(\mathcal{D})}$ is bounded uniformly in $k$ and in $s \in[t, T]$. Moreover, since $p^{k}$ is a solution to equation (3.2) in the sense of Definition 3.1 it holds, from (3.3)

$$
\partial_{t}\left\|p_{s}^{k}\right\|_{\mathbb{L}^{2}(\mathcal{D})}^{2} \leq-\left\|\nabla p_{s}^{k}\right\|_{\mathbb{L}^{2}(\mathcal{D})}^{2}+K\left\|p_{s}^{k}\right\|_{\mathbb{L}^{2}(\mathcal{D})}^{2},
$$

so that $\left(p^{k}\right)_{k \in \mathbb{N}}$ is also bounded in $\mathbb{L}^{2}\left((t, T), \mathbb{H}^{1}(\mathcal{D})\right)$. Adapting the proof of Lemma 4.8, we find that the family $\left(p_{\mid \mathcal{O}}^{k}\right)$ is compact in $\mathbb{L}^{2}\left((t, T), \mathbb{L}^{2}(\mathcal{O})\right)$ for any open subset $\mathcal{O}$ of $\mathcal{D}$. By a diagonal argument, and using the proof of Lemma 4.9 we can thus construct a subsequence $k_{n}$ such that:

- $P^{k_{n}}$ converges to a probability measure $P^{0}$ whose time marginals $P_{t}$ have a density $p_{t}^{0}$, for all $t>0$;

- $p^{k_{n}}$ converges almost everywhere on $(0, T) \times \mathcal{D}$ and in $\mathbb{L}^{1}((0, T) \times \mathcal{D})$ to $p^{0}$;

- $\frac{p^{k_{n}, \partial_{1} V}}{p^{k_{n}, 1}}$ converges almost everywhere on $(0, T) \times \mathcal{D}$ to $\frac{p^{0, \partial_{1} V}}{p^{0,1}}$.

Then, adapting the proof of Theorem 4.5, we see that $P^{0}$ solves the martingale problem. 


\subsection{Rate of convergence}

We are going to exhibit a control on the rate of the convergence of $p^{\eta}$ to $p$. Moreover, we give an estimate of the difference between $\frac{\varphi_{\eta} * p^{\eta, \partial_{1} V}}{\varphi_{\eta} * p^{\eta, 1}}$ and the biasing force $A_{t}^{\prime}=\frac{p_{t}^{\partial_{1} V}}{p_{t}^{1}}$ which is the quantity one is interested in practice.

Theorem 4.11. Under Assumptions i and ii, it holds, in the compact case,

$$
\left\|p^{\eta}-p\right\|_{\mathbb{L}^{\infty}\left((0, T), \mathbb{L}^{2}\left(\mathbb{T}^{d}\right)\right)}+\left\|p^{\eta}-p\right\|_{\mathbb{L}^{2}\left((0, T), \mathbb{H}^{1}\left(\mathbb{T}^{d}\right)\right)} \leq K(\alpha+\sqrt{\varepsilon}),
$$

and, in the non compact case, under the additional Assumption iii

$$
\left\|p^{\eta}-p\right\|_{\mathbb{L}^{\infty}\left((0, T), \mathbb{L}^{2}(w)\right)}+\left\|p^{\eta}-p\right\|_{\mathbb{L}^{2}\left((0, T), \mathbb{H}^{1}(w)\right)} \leq K(\alpha+\sqrt{\varepsilon})
$$

for some positive constant $K$ not depending on $\alpha$ and $\varepsilon$. Moreover, we have the following bound on the estimation of the biasing force:

$$
\left\|\frac{\varphi_{\eta} * p_{t}^{\eta, \partial_{1} V}}{\varphi_{\eta} * p_{t}^{\eta, 1}}-\frac{p_{t}^{\partial_{1} V}}{p_{t}^{1}}\right\|_{\mathbb{L}^{2}\left((0, T), \mathbb{L}^{\infty}(\mathbb{T})\right)} \leq K(\alpha+\sqrt{\varepsilon})
$$

Proof. We give the proof in the non compact case, the one in the compact case being very similar. Similar calculations as in the proof of Theorem 3.6 yield:

$$
\begin{aligned}
\frac{1}{2} \partial_{t}\left\|p_{t}-p_{t}^{\eta}\right\|_{\mathbb{L}^{2}(w)}^{2}+\left\|\nabla p_{t}-\nabla p_{t}^{\eta}\right\|_{\mathbb{L}^{2}(w)}^{2} \leq & K\left\|p_{t}-p_{t}^{\eta}\right\|_{\mathbb{L}^{2}(w)}\left\|\nabla p_{t}-\nabla p_{t}^{\eta}\right\|_{\mathbb{L}^{2}(w)}+K\left\|p_{t}-p_{t}^{\eta}\right\|_{\mathbb{L}^{2}(w)}^{2} \\
& +\left\|\nabla p_{t}^{\eta}-\nabla p_{t}\right\|_{\mathbb{L}^{2}(w)}\left\|p_{t} \frac{p_{t}^{\partial_{1} V}}{p_{t}^{1}}-p_{t}^{\eta} \frac{p_{t}^{\eta, \partial_{1} V} * \varphi_{\eta}}{p_{t}^{\eta, 1} * \varphi_{\eta}}\right\|_{\mathbb{L}^{2}(w)}
\end{aligned}
$$

We now estimate the last term:

$$
\begin{aligned}
\left\|p_{t} \frac{p_{t}^{\partial_{1} V}}{p_{t}^{1}}-p_{t}^{\eta} \frac{p_{t}^{\eta, \partial_{1} V} * \varphi_{\eta}}{p_{t}^{\eta, 1} * \varphi_{\eta}}\right\|_{\mathbb{L}^{2}(w)} \leq & \left\|\frac{p_{t}}{p_{t}^{1}}\left(p_{t}^{\partial_{1} V}-\varphi_{\eta} * p_{t}^{\eta, \partial_{1} V}\right)\right\|_{\mathbb{L}^{2}(w)}+\left\|p_{t} \varphi_{\eta} * p_{t}^{\eta, \partial_{1} V}\left(\frac{1}{p_{t}^{1}}-\frac{1}{\varphi_{\eta} * p_{t}^{\eta, 1}}\right)\right\|_{\mathbb{L}^{2}(w)} \\
& +\left\|\left(p_{t}-p_{t}^{\eta}\right) \frac{\varphi_{\eta} * p_{t}^{\eta, \partial_{1} V}}{\varphi_{\eta} * p_{t}^{\eta, 1}}\right\|_{\mathbb{L}^{2}(w)} \\
\leq & \left\|p_{t}\right\|_{\mathbb{L}^{2}(w)}\left\|\frac{1}{p_{t}^{1}}\left(p_{t}^{\partial_{1} V}-\varphi_{\eta} * p_{t}^{\eta, \partial_{1} V}\right)\right\|_{\mathbb{L}^{\infty}(\mathbb{T})} \\
& +\left\|p_{t}\right\|_{\mathbb{L}^{2}(w)}\left\|\frac{\varphi_{\eta} * p_{t}^{\eta, \partial_{1} V}}{p_{t}^{1}\left(\varphi_{\eta} * p_{t}^{\eta, 1}\right)}\left(\varphi_{\eta} * p_{t}^{\eta, 1}-p_{t}^{1}\right)\right\|_{\mathbb{L}^{\infty}(\mathbb{T})} \\
& +\left\|\left(p_{t}-p_{t}^{\eta}\right) \frac{\varphi_{\eta} * p_{t}^{\eta, \partial_{1} V}}{\varphi_{\eta} * p_{t}^{\eta, 1}}\right\|_{\mathbb{L}^{2}(w)}
\end{aligned}
$$

From Lemma 2.2, $p_{t}^{1}$ is bounded from below uniformly in time. Using this together with the facts that $\partial_{1} V$ is bounded and $p \in \mathbb{L}^{\infty}\left((0, T), \mathbb{L}^{2}(w)\right)$, one obtains

$$
\left\|p_{t} \frac{p_{t}^{\partial_{1} V}}{p_{t}^{1}}-p_{t}^{\eta} \frac{p_{t}^{\eta, \partial_{1} V} * \varphi_{\eta}}{p_{t}^{\eta, 1} * \varphi_{\eta}}\right\|_{\mathbb{L}^{2}(w)} \leq K\left(\left\|p_{t}^{\partial_{1} V}-\varphi_{\eta} * p_{t}^{\eta, \partial_{1} V}\right\|_{\mathbb{L}^{\infty}(\mathbb{T})}+\left\|p_{t}^{1}-\varphi_{\eta} * p_{t}^{\eta, 1}\right\|_{\mathbb{L}^{\infty}(\mathbb{T})}+\left\|p_{t}-p_{t}^{\eta}\right\|_{\mathbb{L}^{2}(w)}\right)
$$


Consequently, we have to estimate $\left\|p_{t}^{1}-\varphi_{\eta} * p_{t}^{\eta, 1}\right\|_{\mathbb{L}^{\infty}(\mathbb{T})}$ and $\left\|p_{t}^{\partial_{1} V}-\varphi_{\eta} * p_{t}^{\eta, \partial_{1} V}\right\|_{\mathbb{L}^{\infty}(\mathbb{T})}$. It holds, for $\gamma \in(0,1 / 2)$,

$$
\begin{aligned}
\left\|p_{t}^{\partial_{1} V}-\varphi_{\eta} * p_{t}^{\eta, \partial_{1} V}\right\|_{\mathbb{L}^{\infty}(\mathbb{T})} & \leq\left\|\varphi_{\eta} *\left(p_{t}^{\partial_{1} V}-p_{t}^{\eta, \partial_{1} V}\right)\right\|_{\mathbb{L}^{\infty}(\mathbb{T})}+\left\|p_{t}^{\partial_{1} V}-\varphi_{\eta} * p_{t}^{\partial_{1} V}\right\|_{\mathbb{L}^{\infty}(\mathbb{T})} \\
& \leq K \alpha+\left\|p_{t}^{\partial_{1} V}-p_{t}^{\eta, \partial_{1} V}\right\|_{\mathbb{L}^{\infty}(\mathbb{T})}+\left\|p_{t}^{\partial_{1} V}-\varphi_{\eta} * p_{t}^{\partial_{1} V}\right\|_{\mathbb{L}^{\infty}(\mathbb{T})} \\
& \leq K\left(\alpha+\left\|p_{t}^{\partial_{1} V}-p_{t}^{\eta, \partial_{1} V}\right\|_{\mathbb{H}^{1}(\mathbb{T})}^{1 / 2+\gamma}\left\|p_{t}^{\partial_{1} V}-p_{t}^{\eta, \partial_{1} V}\right\|_{\mathbb{L}^{2}(\mathbb{T})}^{1 / 2-\gamma}\right)+\left\|p_{t}^{\partial_{1} V}-\varphi_{\eta} * p_{t}^{\partial_{1} V}\right\|_{\mathbb{L}^{\infty}(\mathbb{T})} \\
& \leq K\left(\alpha+\left\|p_{t}-p_{t}^{\eta}\right\|_{\mathbb{H}^{1}(w)}^{1 / 2+\gamma}\left\|p_{t}-p_{t}^{\eta}\right\|_{\mathbb{L}^{2}(\mathbb{T})}^{1 / 2-\gamma}\right)+\left\|p_{t}^{\partial_{1} V}-\varphi_{\eta} * p_{t}^{\partial_{1} V}\right\|_{\mathbb{L}^{\infty}(\mathbb{T})} .
\end{aligned}
$$

Likewise, we have

$$
\left\|p_{t}^{1}-\varphi_{\eta} * p_{t}^{\eta, 1}\right\|_{\mathbb{L}^{\infty}(\mathbb{T})} \leq K \alpha+K\left\|p_{t}-p_{t}^{\eta}\right\|_{\mathbb{H}^{1}(w)}^{1 / 2+\gamma}\left\|p_{t}-p_{t}^{\eta}\right\|_{\mathbb{L}^{2}(\mathbb{T})}^{1 / 2-\gamma}+\left\|p_{t}^{1}-\varphi_{\eta} * p_{t}^{1}\right\|_{\mathbb{L}^{\infty}(\mathbb{T})} .
$$

To conclude, notice that, in view of Lemma 1.1, $p_{t}^{\partial_{1} V}$ lies in $\mathbb{H}^{1}(\mathbb{T})$. Thus $p_{t}^{\partial_{1} V}$ is Hölder continuous with

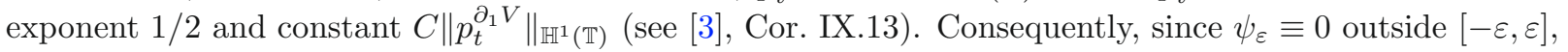

$$
\begin{aligned}
\left|p_{t}^{\partial_{1} V}(x)-\varphi_{\eta} * p_{t}^{\partial_{1} V}(x)\right| & =\left|\alpha \int_{\mathbb{T}} p_{t}^{\partial_{1} V}(x) \mathrm{d} x+\int_{\mathbb{T}} \psi_{\varepsilon}(y)\left(p_{t}^{\partial_{1} V}(x)-p_{t}^{\partial_{1} V}(x-y)\right) \mathrm{d} y\right| \\
& \leq K\left(\alpha+\left\|p_{t}^{\partial_{1} V}\right\|_{\mathbb{H}^{1}(\mathbb{T})} \int_{\mathbb{T}} \psi_{\varepsilon}(y) \sqrt{y} \mathrm{~d} y\right) \\
& \leq K\left(\alpha+\left\|p_{t}^{\partial_{1} V}\right\|_{\mathbb{H}^{1}(\mathbb{T})} \sqrt{\varepsilon} \int_{\mathbb{T}} \psi_{\varepsilon}(y) \mathrm{d} y\right) \\
& \leq K\left(\alpha+\sqrt{\varepsilon}\left\|p_{t}\right\|_{\mathbb{H}^{1}(w)}\right) .
\end{aligned}
$$

The same inequality holds for $p^{1}$

$$
\left|p_{t}^{1}(x)-\varphi_{\eta} * p_{t}^{1}(x)\right| \leq K\left(\alpha+\sqrt{\varepsilon}\left\|p_{t}\right\|_{\mathbb{H}^{1}(w)}\right) .
$$

Gathering all the previous inequalities, we obtain,

$$
\begin{aligned}
\frac{1}{2} \partial_{t}\left\|p_{t}-p_{t}^{\eta}\right\|_{\mathbb{L}^{2}(w)}^{2}+\left\|\nabla p_{t}-\nabla p_{t}^{\eta}\right\|_{\mathbb{L}^{2}(w)}^{2} \leq & K\left\|p_{t}-p_{t}^{\eta}\right\|_{\mathbb{L}^{2}(w)}\left\|\nabla p_{t}-\nabla p_{t}^{\eta}\right\|_{\mathbb{L}^{2}(w)}+K\left\|p_{t}-p_{t}^{\eta}\right\|_{\mathbb{L}^{2}(w)}^{2} \\
& +K\left\|p_{t}-p_{t}^{\eta}\right\|_{\mathbb{H}^{1}(w)}^{1 / 2+\gamma}\left\|p_{t}-p_{t}^{\eta}\right\|_{\mathbb{L}^{2}(\mathbb{T})}^{1 / 2-\gamma}\left\|\nabla p_{t}-\nabla p_{t}^{\eta}\right\|_{\mathbb{L}^{2}(w)} \\
& +K\left(\alpha+\sqrt{\varepsilon}\left\|p_{t}\right\|_{\mathbb{H}^{1}(w)}\right)\left\|\nabla p_{t}-\nabla p_{t}^{\eta}\right\|_{\mathbb{L}^{2}(w)} \\
& +K \alpha\left\|\nabla p_{t}-\nabla p_{t}^{\eta}\right\|_{\mathbb{L}^{2}(w)}
\end{aligned}
$$

Consequently, from Young's inequality,

$$
\partial_{t}\left\|p_{t}-p_{t}^{\eta}\right\|_{\mathbb{L}^{2}(w)}^{2}+\left\|\nabla p_{t}^{\eta}-\nabla p_{t}\right\|_{\mathbb{L}^{2}(w)}^{2} \leq K\left(\left\|p_{t}-p_{t}^{\eta}\right\|_{\mathbb{L}^{2}(w)}^{2}+\alpha^{2}+\varepsilon\left\|p_{t}\right\|_{\mathbb{H}^{1}(w)}^{2}\right) .
$$

Grönwall's Lemma yields the first statement of Theorem 4.11, noticing that $p$ lies in $\mathbb{L}^{2}\left((0, T), \mathbb{H}^{1}(w)\right)$.

For the second statement, arguing as we did above, it holds that

$$
\begin{aligned}
&\left\|\frac{\varphi_{\eta} * p_{t}^{\eta, \partial_{1} V}}{\varphi_{\eta} * p_{t}^{\eta, 1}}-\frac{p_{t}^{\partial_{1} V}}{p_{t}^{1}}\right\|_{\mathbb{L}^{\infty}(\mathbb{T})} \leq K\left(\left\|p_{t}^{1}-\varphi_{\eta} * p_{t}^{\eta, 1}\right\|_{\mathbb{L}^{\infty}(\mathbb{T})}+\left\|\varphi_{\eta} * p_{t}^{\eta, \partial_{1} V}-p_{t}^{\partial_{1} V}\right\|_{\mathbb{L}^{\infty}(\mathbb{T})}\right) \\
& \leq K\left(\alpha+\sqrt{\varepsilon}\left\|p_{t}\right\|_{\mathbb{H}^{1}(\mathbb{T})}+\left\|p_{t}-p_{t}^{\eta}\right\|_{\mathbb{H}^{1}(w)}\right) .
\end{aligned}
$$

We finish the proof by squaring this inequality and then integrating. 


\section{AN interaCting PARTICLE SYSTEM APPROXIMATION}

In this section, we prove the convergence of the interacting particle system to the regularized nonlinear processes, and we estimate the difference between the regularized biasing force $\frac{\varphi_{\eta} * p_{t}^{\eta, \partial_{1} V}}{\varphi_{\eta} * p_{t}^{\eta, 1}}$ and its particle approximation.

Theorem 5.1. Let $T$ be a positive time. Under Assumptions i, ii, iv and $\mathrm{v}$, the solution $\left(X_{t, n, N}^{\eta}\right)_{N \geq 1}$ of (2.3) with initial condition $X_{0, n, N}^{\eta}=X_{0, n}$ converges to the solution $\bar{X}_{t, n}^{\eta}$ to (4.1) with initial condition $X_{0, n}$ in the following sense: for all $1 \leq n \leq N$, and for $\varepsilon, \alpha \leq 1$,

$$
\mathbb{E}\left[\sup _{t \in[0, T]}\left|\bar{X}_{t, n}^{\eta}-X_{t, n, N}^{\eta}\right|\right]<\frac{1}{\sqrt{N}} \mathrm{e}^{\frac{K}{\alpha \varepsilon^{2}}}
$$

$K$ being some constant not depending on $\alpha, \varepsilon$ and $N$.

Moreover, one has

$$
\mathbb{E}\left[\sup _{t \in[0, T], x^{1} \in \mathbb{T}}\left|\frac{\varphi_{\eta} * p^{\eta, \partial_{1} V}}{\varphi_{\eta} * p^{\eta, 1}}\left(x^{1}\right)-\frac{\sum_{n=1}^{N} \varphi_{\eta}\left(x^{1}-X_{t, n, N}^{\eta, 1}\right) \partial_{1} V\left(X_{t, n, N}^{\eta}\right)}{\sum_{n=1}^{N} \varphi_{\eta}\left(x^{1}-X_{t, n, N}^{\eta, 1}\right)}\right|\right] \leq \frac{1}{\sqrt{N}} \mathrm{e}^{\frac{K}{\alpha \varepsilon^{2}}} .
$$

Notice that the right hand side of (5.1) explodes when $\varepsilon$ goes to 0 for a fixed value of $N$, so that the size of $\varepsilon$ has to be chosen carefully depending on the value of $N$. We will also investigate this point numerically in the next section.

To simplify notation, we omit the subscript $N$ and the superscript $\eta$. We first establish the following inequality:

Lemma 5.2. We have, for $\varepsilon, \alpha<1$, and for any $t$ in $(0, T]$,

$$
\left|X_{t, n}-\bar{X}_{t, n}\right| \leq \frac{K}{\alpha \varepsilon^{2}} \int_{0}^{t}\left(\left|X_{s, n}-\bar{X}_{s, n}\right|+\frac{1}{N} \sum_{m=1}^{N}\left|X_{s, m}-\bar{X}_{s, m}\right|\right) \mathrm{d} s+K \int_{0}^{t} A_{s}^{n, N} \mathrm{~d} s
$$

where $K$ does not depend on $\alpha, \varepsilon$ and $t$, and $A_{t}^{n, N}$ is defined by

$$
\begin{aligned}
A_{t}^{n, N}= & \frac{1}{\alpha}\left(\left|\frac{1}{N} \sum_{m=1}^{N} \varphi_{\eta}\left(\bar{X}_{s, n}^{1}-\bar{X}_{s, m}^{1}\right) \partial_{1} V\left(\bar{X}_{s, m}\right)-\varphi_{\eta} * p_{s}^{\eta, \partial_{1} V}\left(\bar{X}_{s, n}^{1}\right)\right|\right. \\
& \left.+\left|\frac{1}{N} \sum_{m=1}^{N} \varphi_{\eta}\left(\bar{X}_{s, n}^{1}-\bar{X}_{s, m}^{1}\right)-\varphi_{\eta} * p_{s}^{\eta, 1}\left(\bar{X}_{s, n}^{1}\right)\right|\right) .
\end{aligned}
$$

Proof. From the definition of $X_{t, n}$ and $\bar{X}_{t, n}$, we have

$$
\begin{aligned}
\left|X_{t, n}-\bar{X}_{t, n}\right| \leq & \left|\int_{0}^{t}\left(\nabla V\left(X_{s, n}\right)-\nabla V\left(\bar{X}_{s, n}\right)\right) \mathrm{d} s\right| \\
& +\left|\int_{0}^{t} \frac{\sum_{m=1}^{N} \varphi_{\eta}\left(X_{s, n}^{1}-X_{s, m}^{1}\right) \partial_{1} V\left(X_{s, m}\right)}{\sum_{m=1}^{N} \varphi_{\eta}\left(X_{s, n}^{1}-X_{s, m}^{1}\right)} \mathrm{d} s-\int_{0}^{t} \frac{\varphi_{\eta} * p_{s}^{\eta, \partial_{1} V}\left(\bar{X}_{s, n}^{1}\right)}{\varphi_{\eta} * p_{s}^{\eta, 1}\left(\bar{X}_{s, n}^{1}\right)} \mathrm{d} s\right| .
\end{aligned}
$$


First, $\left|\int_{0}^{t}\left(\nabla V\left(X_{s, n}\right)-\nabla V\left(\bar{X}_{s, n}\right)\right) \mathrm{d} s\right|$ is bounded from above by $K \int_{0}^{t}\left|X_{s, n}-\bar{X}_{s, n}\right| \mathrm{d} s$, since $\nabla V$ is Lipschitz continuous. Now, we decompose

$$
\begin{aligned}
& \left|\frac{\sum_{m=1}^{N} \varphi_{\eta}\left(X_{s, n}^{1}-X_{s, m}^{1}\right) \partial_{1} V\left(X_{s, m}\right)}{\sum_{m=1}^{N} \varphi_{\eta}\left(X_{s, n}^{1}-X_{s, m}^{1}\right)}-\frac{\varphi_{\eta} * p_{s}^{\eta, \partial_{1} V}\left(\bar{X}_{s, n}^{1}\right)}{\varphi_{\eta} * p_{s}^{\eta, 1}\left(\bar{X}_{s, n}^{1}\right)}\right| \\
& \leq\left|\frac{\sum_{m=1}^{N} \varphi_{\eta}\left(X_{s, n}^{1}-X_{s, m}^{1}\right) \partial_{1} V\left(X_{s, m}\right)}{\sum_{m=1}^{N} \varphi_{\eta}\left(X_{s, n}^{1}-X_{s, m}^{1}\right)}-\frac{\sum_{m=1}^{N} \varphi_{\eta}\left(\bar{X}_{s, n}^{1}-\bar{X}_{s, m}^{1}\right) \partial_{1} V\left(\bar{X}_{s, m}\right)}{\sum_{m=1}^{N} \varphi_{\eta}\left(\bar{X}_{s, n}^{1}-\bar{X}_{s, m}^{1}\right)}\right| \\
& \quad+\left|\frac{\sum_{m=1}^{N} \varphi_{\eta}\left(\bar{X}_{s, n}^{1}-\bar{X}_{s, m}^{1}\right) \partial_{1} V\left(\bar{X}_{s, m}\right)}{\sum_{m=1}^{N} \varphi_{\eta}\left(\bar{X}_{s, n}^{1}-\bar{X}_{s, m}^{1}\right)}-\frac{\varphi_{\eta} * p_{s}^{\eta, \partial_{1} V}\left(\bar{X}_{s, n}^{1}\right)}{\varphi_{\eta} * p_{s}^{\eta, 1}\left(\bar{X}_{s, n}^{1}\right)}\right| .
\end{aligned}
$$

Using Assumptions i and $\mathrm{v}$, the first term in the right hand-side of (5.2) can be bounded by $\frac{K}{\alpha \varepsilon^{2}}\left(\left|X_{s, n}-\bar{X}_{s, n}\right|+\right.$ $\left.\frac{1}{N} \sum_{m=1}^{N}\left|X_{s, m}-\bar{X}_{s, m}\right|\right)$, and the second term in the right hand side of (5.2) can be bounded by $K A_{t}^{n, N}$.

Proof of Theorem 5.1. As a consequence of Lemma 5.2, we get, for $\alpha, \varepsilon \leq 1$,

$$
\sup _{t \in[0, T]}\left|X_{t, n}-\bar{X}_{t, n}\right| \leq \frac{K}{\alpha \varepsilon^{2}} \int_{0}^{T}\left(\sup _{s \in[0, t]}\left|X_{s, n}-\bar{X}_{s, n}\right|+\frac{1}{N} \sum_{m=1}^{N} \sup _{s \in[0, t]}\left|X_{s, m}-\bar{X}_{s, m}\right|\right) \mathrm{d} t+K \int_{0}^{T} A_{t}^{n, N} \mathrm{~d} t .
$$

Taking the expectation, and using the exchangeability of the couples $\left(X_{n}, \bar{X}_{n}\right)_{1 \leq n \leq N}$, we get

$$
\mathbb{E}\left[\sup _{t \in[0, T]}\left|X_{t, n}-\bar{X}_{t, n}\right|\right] \leq \frac{K}{\alpha \varepsilon^{2}} \int_{0}^{T} \mathbb{E}\left[\sup _{s \in[0, t]}\left|\bar{X}_{s, n}-X_{s, n}\right|\right] \mathrm{d} t+K \int_{0}^{T} \mathbb{E}\left[A_{t}^{n, N}\right] \mathrm{d} t .
$$

By Grönwall's lemma, one obtains

$$
\mathbb{E}\left[\sup _{t \in[0, T]}\left|X_{t, n}-\bar{X}_{t, n}\right|\right] \leq K \mathrm{e}^{\frac{K}{\alpha \varepsilon^{2}} T} \int_{0}^{T} \mathbb{E}\left[A_{t}^{n, N}\right] \mathrm{d} t .
$$

To conclude, we estimate $\int_{0}^{T} \mathbb{E}\left[A_{t}^{n, N}\right] \mathrm{d} t$. Let

$$
\Phi_{t}^{m}=\varphi_{\eta}\left(\bar{X}_{t, 1}^{1}-\bar{X}_{t, m}^{1}\right) \partial_{1} V\left(\bar{X}_{t, m}\right)-\varphi_{\eta} * p_{t}^{\eta, \partial_{1} V}\left(\bar{X}_{t, 1}^{1}\right)
$$

and

$$
\Psi_{t}^{m}=\varphi_{\eta}\left(\bar{X}_{t, 1}^{1}-\bar{X}_{t, m}^{1}\right)-\varphi_{\eta} * p_{t}^{\eta, 1}\left(\bar{X}_{t, 1}^{1}\right)
$$

We have, for $t \leq T$, using again the exchangeability of the couples $\left(X_{n}, \bar{X}_{n}\right)_{1 \leq n \leq N}$,

$$
\begin{aligned}
{\left[\mathbb{E} A_{t}^{n, N}\right]^{2} } & \leq \mathbb{E}\left[\left(A_{t}^{n, N}\right)^{2}\right] \leq \frac{K}{\alpha^{2}}\left(\mathbb{E}\left[\left(\frac{1}{N} \sum_{m=1}^{N} \Phi_{t}^{m}\right)^{2}\right]+\mathbb{E}\left[\left(\frac{1}{N} \sum_{m=1}^{N} \Psi_{t}^{m}\right)^{2}\right]\right) \\
& =\frac{K}{N^{2} \alpha^{2}} \sum_{m, m^{\prime}}\left(\mathbb{E}\left[\Phi_{t}^{m} \Phi_{t}^{m^{\prime}}\right]+\mathbb{E}\left[\Psi_{t}^{m} \Psi_{t}^{m^{\prime}}\right]\right) .
\end{aligned}
$$

But the $\Phi_{t}^{m}$ and $\Psi_{t}^{m}$ are centered for $m \geq 2$, and, for $m \neq m^{\prime}, \Phi_{t}^{m}$ and $\Phi_{t}^{m^{\prime}}$ (as well as $\Psi_{t}^{m}$ and $\Psi_{t}^{m^{\prime}}$ ) are independent conditionally on $\bar{X}_{t, 1}$. Therefore the double products vanish, and, by exchangeability

$$
\left[\mathbb{E} A_{t}^{n, N}\right]^{2} \leq \frac{K(N-1)}{\alpha^{2} N^{2}}\left(\mathbb{E}\left[\left(\Phi_{t}^{2}\right)^{2}\right]+\mathbb{E}\left[\left(\Psi_{t}^{2}\right)^{2}\right]\right)+\frac{K}{\alpha^{2} N^{2}}\left(\mathbb{E}\left[\left(\Phi_{t}^{1}\right)^{2}\right]+\mathbb{E}\left[\left(\Psi_{t}^{1}\right)^{2}\right]\right) .
$$


But one has $\mathbb{E}\left[\left(\Phi_{t}^{2}\right)^{2}\right]+\mathbb{E}\left[\left(\Psi_{t}^{2}\right)^{2}\right] \leq K \varepsilon^{-2}$ and $\mathbb{E}\left[\left(\Phi_{t}^{1}\right)^{2}\right]+\mathbb{E}\left[\left(\Psi_{t}^{1}\right)^{2}\right] \leq K \varepsilon^{-2}$, and the first assertion in Theorem 5.1 follows.

For the estimation of the force, adapting the proof of Lemma 5.2, we see that

$$
\begin{aligned}
& \mathbb{E}\left[\sup _{t \in[0, T]}\left|\frac{\varphi_{\eta} * p_{t}^{\eta, \partial_{1} V}}{\varphi_{\eta} * p_{t}^{\eta, 1}}\left(x^{1}\right)-\frac{\sum_{n=1}^{N} \varphi_{\eta}\left(x^{1}-X_{t, n, N}^{1}\right) \partial_{1} V\left(X_{t, n, N}\right)}{\sum_{n=1}^{N} \varphi_{\eta}\left(x^{1}-X_{t, n, N}^{1}\right)}\right|\right] \\
\leq & \frac{1}{\alpha} \mathbb{E}\left[\sup _{t \in[0, T]}\left|\frac{1}{N} \sum_{n=1}^{N} \varphi_{\eta}\left(x^{1}-\bar{X}_{t, n}^{1}\right) \partial_{1} V\left(\bar{X}_{t, n}\right)-\varphi_{\eta} * p_{t}^{\eta, \partial_{1} V}\left(x^{1}\right)\right|\right] \\
& \quad+\frac{1}{\alpha} \mathbb{E}\left[\sup _{t \in[0, T]}\left|\frac{1}{N} \sum_{n=1}^{N} \varphi_{\eta}\left(x^{1}-\bar{X}_{t, n}^{1}\right)-\varphi_{\eta} * p_{t}^{\eta, 1}\left(x^{1}\right)\right|\right]+\frac{K}{\alpha \varepsilon^{2} N} \mathbb{E}\left[\sup _{t \in[0, T]} \sum_{n=1}^{N}\left|X_{t, n}-\bar{X}_{t, n}\right|\right] \\
\leq & \frac{1}{\sqrt{N}} \mathrm{e}^{\frac{K}{\alpha \varepsilon^{2}}} .
\end{aligned}
$$

Indeed, $\left(\varphi_{\eta}\left(x^{1}-\bar{X}_{t, n}^{1}\right) \partial_{1} V\left(\bar{X}_{t, n}\right)-\varphi_{\eta} * p_{t}^{\eta, \partial_{1} V}\left(x^{1}\right)\right)_{n \in\{1 \ldots N\}}$, as well as $\left(\varphi_{\eta}\left(x^{1}-\bar{X}_{t, n}^{1}\right)-\varphi_{\eta} * p_{t}^{\eta, 1}\left(x^{1}\right)\right)_{n \in\{1 \ldots N\}}$, are i.i.d. centered random variables whose variance is bounded by $\frac{K}{\varepsilon^{2}}$, uniformly in time.

\section{Numerical RESULtS}

In this section we give some numerical simulations to illustrate our previous results. Here, the parameter $\alpha$, which was introduced to enable theoretical estimations, is taken to be 0 .

Notice that the discretization method used here in the simulations and based on a Nadaraya-Watson estimator is different from the classical implementations of the ABF technique [8]. Indeed, in molecular dynamics codes, time averages are used in order to smooth the problem: first the equation on $A_{t}$ given in (1.4) is typically replaced by

$$
\partial_{t} A_{t}^{\prime}(z)=\frac{1}{\tau}\left(\mathbb{E}\left[F\left(X_{t}\right) \mid \xi\left(X_{t}\right)=z\right]-A_{t}\right)
$$

which makes $A_{t}$ vary more smoothly. Second, in addition to the particle approximation, an ergodic average for the computation of the conditional expectation in (2.1) is used.

In order to accelerate the convergence, one can also use a selection mechanism that gives more weight to particles located in less explored areas (see [10]).

\subsection{Efficiency of the ABF method}

Let us introduce a low dimensional example to illustrate the efficiency of the ABF method and its particle approximation.

In this first example, we simulated the particle approximation with 1000 particles, in the potential defined for $(x, y)$ in $[-2,2] \times \mathbb{R}$ by

$$
V_{1}(x, y)=5 \mathrm{e}^{-x^{2}-y^{2}}-5 \mathrm{e}^{-(x-1)^{2}-y^{2}}-5 \mathrm{e}^{-(x+1)^{2}-y^{2}}+0.2 x^{4}+0.2 y^{4},
$$

and extended periodically in the $x$ direction with period 4 . The level sets of $V_{1}$ are depicted in Figure 1 .

In Figure 1, we also plotted the position of the particles after 2000 iteration of an Euler-Maruyama approximation of equation (2.3) with a time step of 0.01 . The value of the parameters are $\varepsilon=0.01, \beta=10$ and $N=1000$. In Figure 2, we plotted the graph of the mean force (computed by numerical integration, which is still possible due to the low dimensionality), and the value of the approximate mean force computed on a regular grid. The $\mathbb{L}^{1}$-distance between the two functions is $6.93 \times 10^{-2}$, while the $\mathbb{L}^{1}$-norm of the function $A^{\prime}$ is 12.9 . 


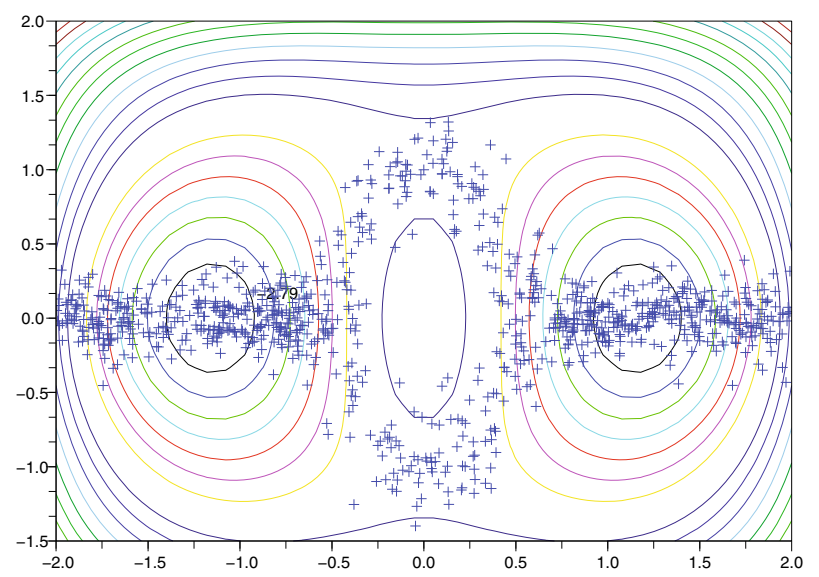

Figure 1. Contour plot of the potential $V_{1}$ with the positions of 1000 particles at time $t=20$.

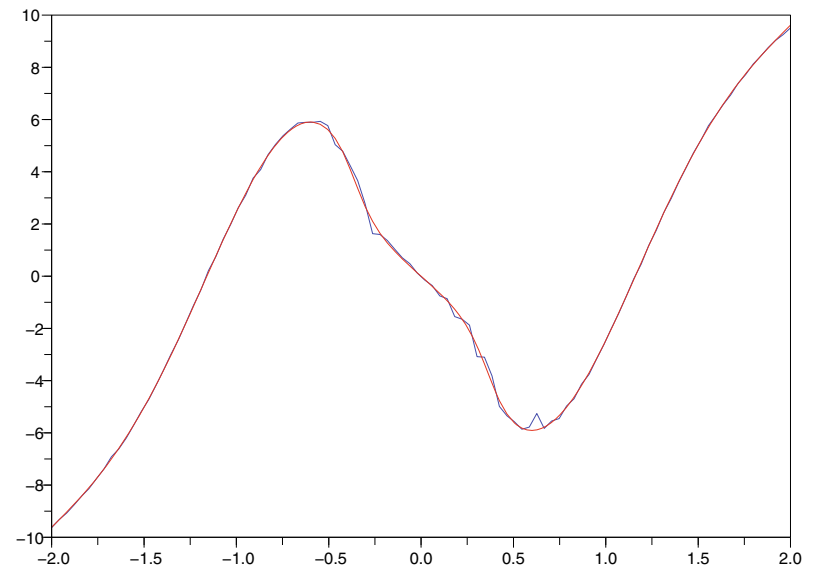

FIgure 2. Particle approximation of the mean force. The smooth curve is the actual value of the mean force, the rough one is the approximation.

Notice that without biasing force, one obtains a very poor sampling, since the particles do not escape from the well they started in: see Figure 3, where we plotted 200 independent simulations of a Langevin dynamics (1.1) using 2000 iterations of an Euler-Maruyama scheme of time step 0.01 .

In Figure 4 we show the $\mathbb{L}^{1}$ distance between the actual value of the mean force $A^{\prime}$ and its approximation at time 20, obtained for one simulation of the system, as a function of the number of particles used in the simulation. Using a least square regression, we find that the slope of the curve is approximatively -0.59 , which matches with the theoretical rate of $N^{-1 / 2}$.

\subsection{Tuning of the parameters}

In Theorem 5.1, we showed that the particle approximation converges as $\varepsilon$ goes to 0 and $N$ goes to infinity, provided that $\varepsilon$ does not go to zero too fast compared to $N$. The practical difficulty that one encounters to apply this result is to choose a good scaling for $\varepsilon$ in term of $N$.

In Figure 5, we can see the $\mathbb{L}^{1}$ error between the mean force and its approximation at time 20, as a function of the parameter $\varepsilon$, using $N=1500$ particles. 


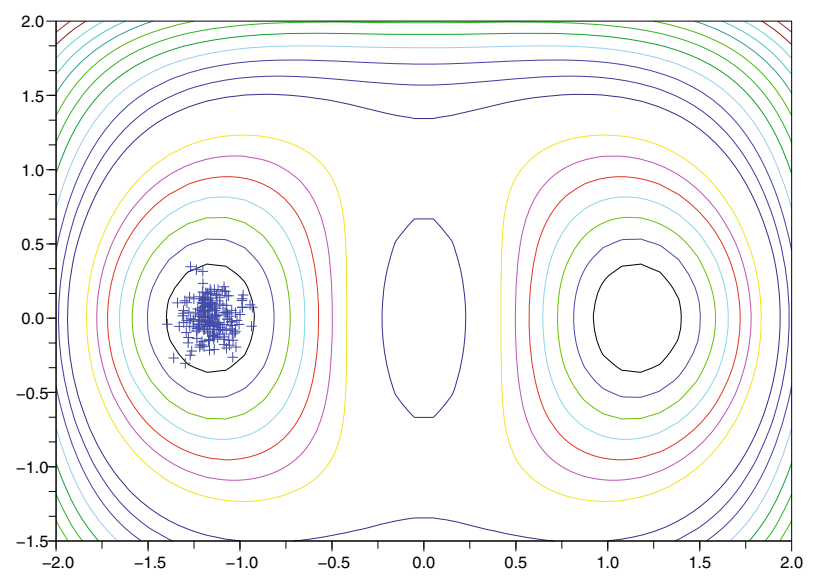

FIGURE 3. 200 independent realizations of a Langevin dynamics at time $t=20$.

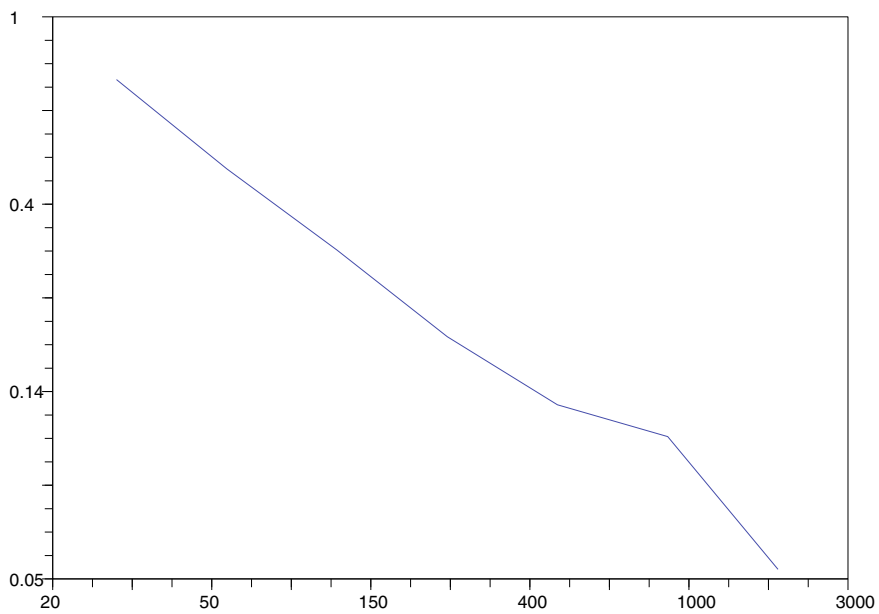

Figure 4. Error as a function of $N$ (logarithmic scale).

Actually, for a fixed value of $N$, there is only a small range of values for $\varepsilon$ for which the error is small.

First, the limit of the error as $\varepsilon$ goes to 0 does not even vanish as $N$ tends to infinity. The reason is that, since the particles interact with each other in a range of $\varepsilon$, the number of particles which interact with a given particle is of order $\varepsilon N$. Hence, when $\varepsilon$ tends to 0 while $N$ is fixed, the particles cannot see each other. Therefore, the natural limit of the particle system in the limit $\varepsilon \rightarrow 0, N$ fixed, should be a system of independent particles following the dynamics

$$
\mathrm{d} X_{t}=\left(-\nabla V\left(X_{t}\right)+e_{1} \partial_{1} V\left(X_{t}\right)\right) \mathrm{d} t+\sqrt{2 \beta^{-1}} \mathrm{~d} W_{t}
$$

Unfortunately, in the general case, the drift in the above dynamics is not obtained as the gradient of a potential, so that no invariant measure for $X_{t}$ is known. This would consequently induce a non vanishing bias in the estimation of $A$. 


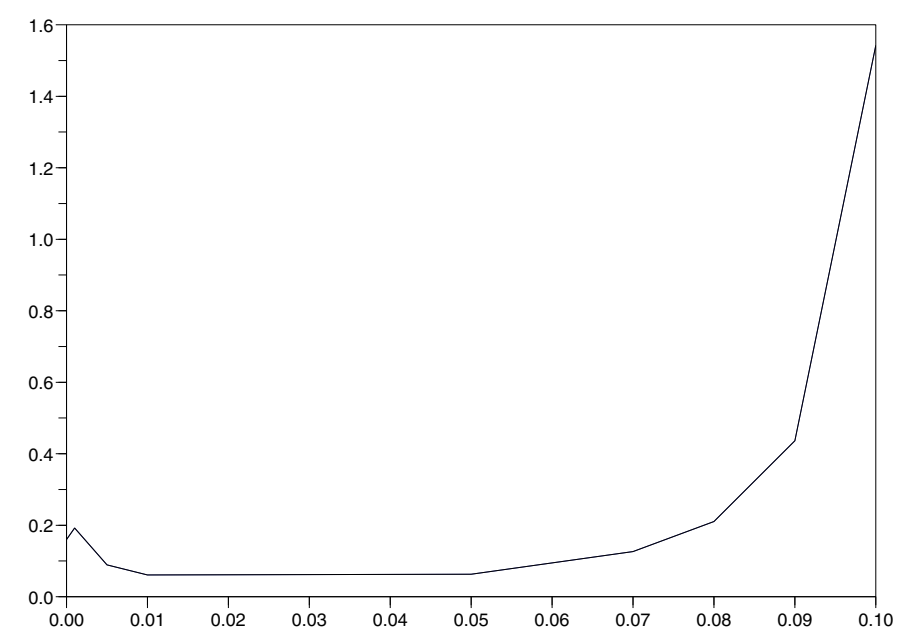

Figure 5. Error as a function of $\varepsilon$.

For example, for the potential $V(x, y)=\frac{1}{2}(y-\sin (2 \pi x))^{2}$, one can prove that the dynamics obtained by canceling the force on the reaction coordinate $x$, namely the couple $\left(\left\{X_{t}\right\}, Y_{t}\right)$ defined by the dynamics

$$
\left\{\begin{array}{l}
\mathrm{d} X_{t}=\sqrt{2} \mathrm{~d} W_{t}^{1}, \\
\mathrm{~d} Y_{t}=\left(-Y_{t}+\sin \left(2 \pi X_{t}\right)\right) \mathrm{d} t+\sqrt{2} \mathrm{~d} W_{t}^{2}
\end{array}\right.
$$

converges in law to the couple $\left(\xi, \int_{0}^{\infty} \mathrm{e}^{-s} \sin \left(2 \pi\left(\xi+\sqrt{2} W_{s}\right)\right) \mathrm{d} s+G\right)$, where $W$ is a standard Brownian motion, $\xi$ is uniformly distributed on $\mathbb{T}$, and $G$ is a standard normal random variable, independent of $W$. This is not the correct limit distribution, since the law of $Y$ conditioned to the value of $\{X\}$ should be Gaussian, which is not the case here.

For a large value of $\varepsilon$, the behavior of the particle system can be really different from the expected behavior of the dynamics (1.4). In the following example, the particles, instead of freely visiting the $x$ axis, keep stuck in the local minima they started in. Indeed, the large value of $\varepsilon$ made that the biasing term is close to the mean of $\partial_{1} V\left(X^{i}\right)$ on all particles, whose value is close to 0 . Consequently, the biasing force is not large enough to prevent the particle from being trapped in the local minima.

In the following example we considered the potential $V_{1}$ defined in (6.1), took $\varepsilon=1$, and simulated 200 particles during 2000 iterations of time step 0.01. The result can be seen in Figure 6.

One way to increase the sample size while keeping the number $N$ of particles fixed is to include time averages for the estimation of the conditional expectation. This is actually the common practice in the applied community $($ see $[4,8])$.

\subsection{Discussion on the choice of the reaction coordinate}

We now give another example to illustrate the limitations of the ABF method. We consider the 4-periodical potential (in the $x$-direction) defined for $(x, y)$ in $[-2,2] \times \mathbb{R}$ by

$$
V_{2}(x, y)=3 \mathrm{e}^{-x^{2}-(y-1 / 3)^{2}}-3 \mathrm{e}^{-x^{2}-(y-5 / 3)^{2}}-5 \mathrm{e}^{-(x-1)^{2}-y^{2}}-5 \mathrm{e}^{-(x+1)^{2}-y^{2}}+0.2 x^{4}+0.2(y-1 / 3)^{4},
$$

whose level sets are depicted in Figure 7. This potential has been introduced in [14].

The potential $V_{2}$ displays two deep minima approximately located at $( \pm 1,0)$. There is a maximum located at $(0,0.5)$, so that there are two possible paths between the main minima. The first one is a direct path meeting 


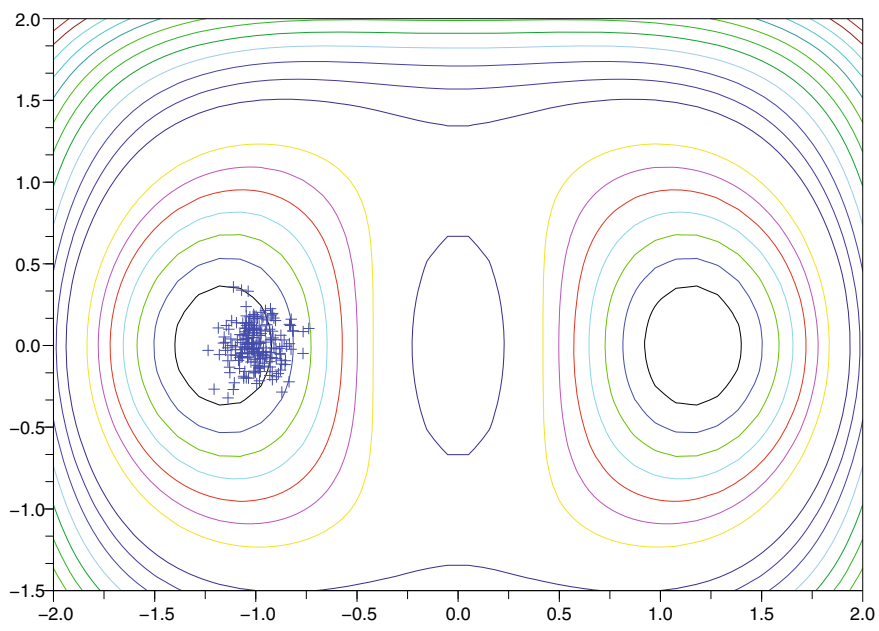

Figure 6 . Bad sampling due to a too large value of $\varepsilon$.

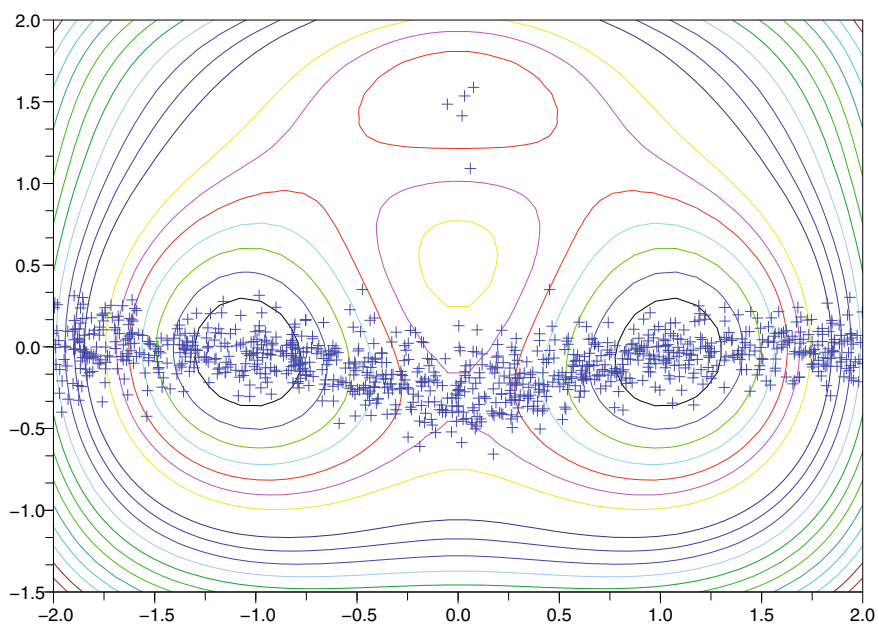

Figure 7. Poor sampling due to a bad choice of the reaction coordinate.

a saddle point approximately at $(0,-0.3)$. The other path goes through two saddle points at $( \pm 0.5,1)$ and a small minima at $(0,1.5)$. Even if the first path is more direct than the second one, the preferred path in low temperature regimes will be the second one, since its energy barrier is smaller.

We simulated the particle approximation of the $\mathrm{ABF}$ method with $N=1000$ particles, window width $\varepsilon=0.01$, after 2000 iterations of an Euler-Maruyama scheme of time step 0.01, and plotted the positions of the particles in Figure 7.

At the low temperature $\beta=10$, the particles are expected to hop from one well to the other mainly through the upper channel, which is not the case here. This is due to a bad choice of the reaction coordinate. Indeed, the biasing force only acts in the $x$ direction, so that a particle trapped in the left side well will naturally escape through a horizontal path, and will take the lower channel. As a result, the computation of the force is clearly biased, because of the poor sampling of the upper channel, see Figure 8 , the $\mathbb{L}^{1}$-distance between the two functions is of 0.4 . 


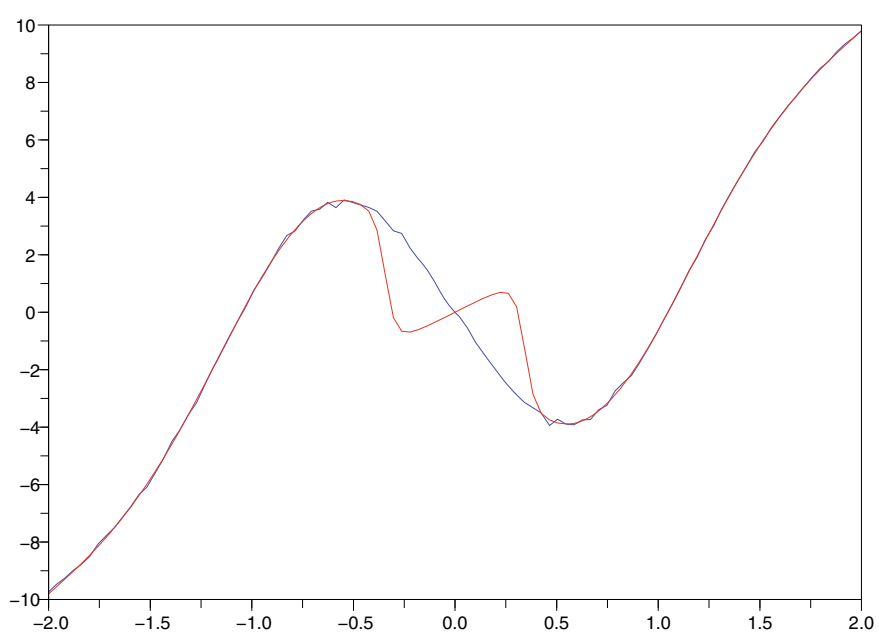

FIGURE 8. Biased evaluation of the biasing force due to a bad choice of the reaction coordinate. The smooth curve is the value of the mean force. The rough curve is the approximation. Here, the approximation does not see the variations of the mean force around 0.

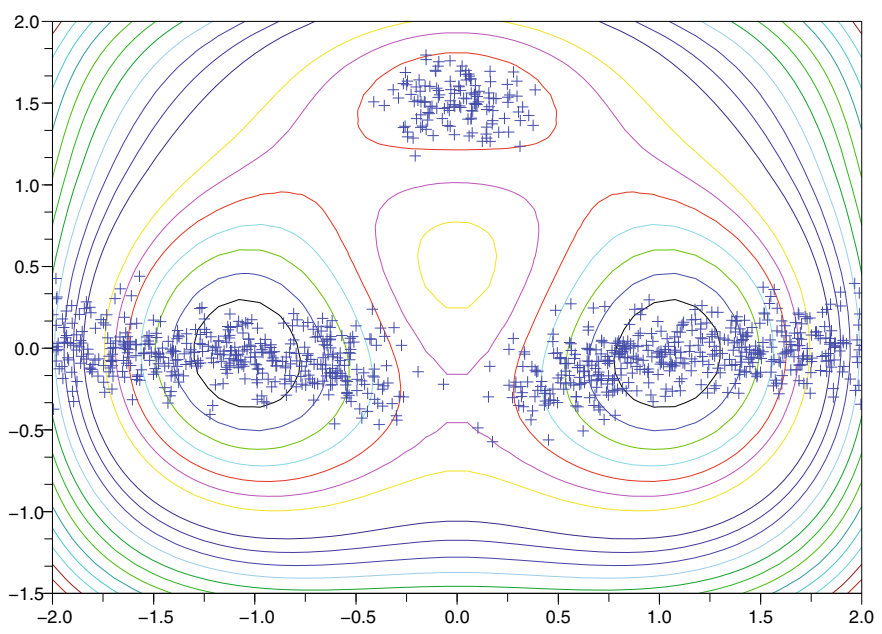

Figure 9. Same simulation as in Figure 7 at time 2000.

We still have convergence to the correct mean force, but at a slow rate, since the reaction coordinate has not been chosen in an optimal way. Indeed, with the same parameters, but after $2 \times 10^{6}$ iterations, the result is much better, see Figures 9 and 10. The $\mathbb{L}^{1}$-distance between the mean force and its approximation is of 0.15 , while the function $A^{\prime}$ has $\mathbb{L}^{1}$-norm 10.9 . 


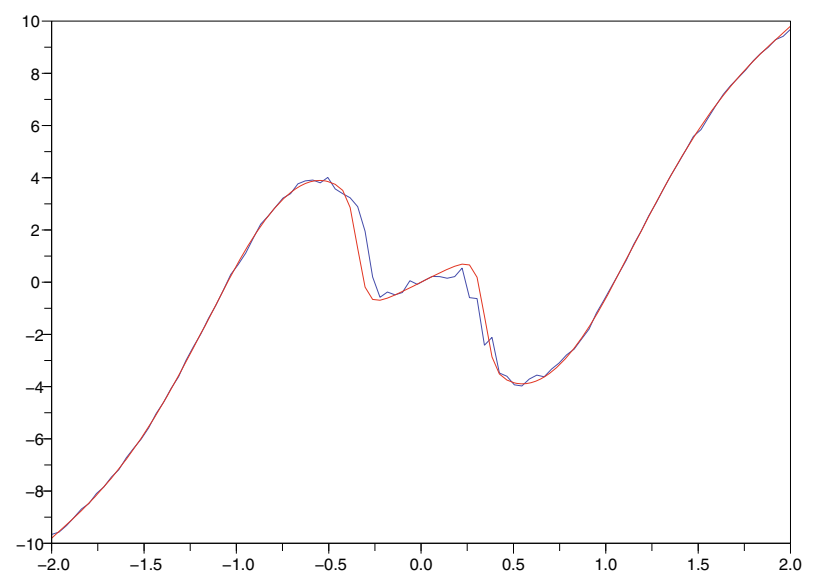

Figure 10. Approximation of the free energy corresponding to Figure 9. The smooth curve is the free energy, the rough one is the approximation.

Acknowledgements. We warmly thank Mathias Rousset for his careful reading of an early version of the manuscript.

\section{REFERENCES}

[1] R. Adams, Sobolev spaces. Academic Press (1978).

[2] M. Bossy, J.F. Jabir and D. Talay, On conditional McKean Lagrangian stochastic models. Prob. Theor. Relat. Fields (to appear).

[3] H. Brézis, Analyse fonctionnelle. Théorie et applications. Collection Mathématiques appliquées pour la maîtrise, Masson, Paris (1983).

[4] C. Chipot and A. Pohorille Eds., Free Energy Calculations, Springer Series in Chemical Physics 86. Springer (2007).

[5] E. Darve and A. Pohorille, Calculating free energy using average forces. J. Chem. Phys. 115 (2001) 9169-9183.

[6] R. Dautray and P.L. Lions, Mathematical Analysis and Numerical Methods for Science and Technology. Springer Verlag (1999).

[7] A. Dermoune, Propagation and conditional propagation of chaos for pressureless gas equations. Prob. Theor. Relat. Fields 126 (2003) 459-479.

[8] J. Hénin and C. Chipot, Overcoming free energy barriers using unconstrained molecular dynamics simulations. J. Chem. Phys. 121 (2004) 2904-2914.

[9] N.V. Krylov and M. Röckner, Strong solutions of stochastic equations with singular time dependent drift. Prob. Theor. Relat. Fields 131 (2005) 154-196.

[10] T. Lelièvre, M. Rousset and G. Stoltz, Computation of free energy profiles with parallel adaptive dynamics. J. Chem. Phys. 126 (2007) 134111.

[11] T. Lelièvre, M. Rousset and G. Stoltz, Long-time convergence of an adaptive biasing force method. Nonlinearity 21 (2008) $1155-1181$

[12] J.L. Lions, Quelques méthodes de résolution des problèmes aux limites non-linéaires. Dunod (1969).

[13] J.L. Lions and E. Magenes, Problèmes aux limites non homogènes et applications. Dunod, Paris (1968-1970).

[14] P. Metzner, C. Schütte and E. Vanden-Eijnden, Illustration of transition path theory on a collection of simple examples. J. Chem. Phys. 125 (2006) 084110.

[15] A.S. Sznitman, Topics in propagation of chaos, Lecture notes in mathematics 1464. Springer-Verlag (1989).

[16] D. Talay and O. Vaillant, A stochastic particle method with random weights for the computation of statistical solutions of McKean-Vlasov equations. Ann. Appl. Prob. 13 (2003) 140-180.

[17] R. Temam, Navier-Stokes equations and nonlinear functionnal analysis. North Holland, Amsterdam (1979).

[18] V.C. Tran, A wavelet particle approximation for McKean-Vlasov and 2D-Navier-Stokes statistical solutions. Stoch. Proc. Appl. 118 (2008) 284-318.

[19] A.B. Tsybakov, Introduction à l'estimation non-paramétrique. Springer (2004). 